\title{
The interplay between decay of the data and regularity of the solution in Schrödinger equations
}

\author{
Alessia Ascanelli ${ }^{1}$ (D) Massimo Cicognani $^{2} \cdot$ Michael Reissig $^{3}$
}

Received: 25 June 2019 / Accepted: 16 November 2019 / Published online: 25 November 2019 (c) Fondazione Annali di Matematica Pura ed Applicata and Springer-Verlag GmbH Germany, part of Springer Nature 2019

\section{Abstract}

We deal with the following Cauchy problem for a Schrödinger equation:

$$
D_{t} u-\Delta u+\sum_{j=1}^{n} a_{j}(t, x) D_{x_{j}} u+b(t, x) u=0, \quad u(0, x)=g(x) .
$$

We assume a decay condition of type $|x|^{-\sigma}, \sigma \in(0,1)$, on the imaginary part of the coefficients $a_{j}$ of the convection term for large values of $|x|$. This condition is known to produce a unique solution with Gevrey regularity of index $s \geq 1$ and loss of an infinite number of derivatives with respect to the data for every $s \leq \frac{1}{1-\sigma}$. In this paper, we consider the case $s>\frac{1}{1-\sigma}$, where, in general, Gevrey ill-posedness holds. We explain how the space where a unique solution exists depends on the decay and regularity of an initial data in $H^{m}, m \geq 0$. As a by-product, we show that a decay condition on data in $H^{m}$ produces a solution with (at least locally) the same regularity as the data, but with an expected different behavior as $|x| \rightarrow \infty$.

Keywords Schrödinger equation · Cauchy problem · Well-posedness $\cdot$ Regularity of solutions · Pseudo-differential operators

Mathematics Subject Classification 35Q41 · 35B65

\section{Introduction and main results}

In this paper, we consider the Cauchy problem

$$
\begin{cases}S u=0, & (t, x) \in[0, T] \times \mathbb{R}^{n} \\ u(0, x)=g(x), & x \in \mathbb{R}^{n}\end{cases}
$$

This research has been supported by INdAM-GNAMPA visiting professors program 2018 and 2019, by the Italian national research fund FFABR 2017 of Alessia Ascanelli and by local research funds FAR 2017 and 2018 of the University of Ferrara.

Alessia Ascanelli

alessia.ascanelli@unife.it

Extended author information available on the last page of the article 
for the operator

$$
S=D_{t}-\Delta+\sum_{j=1}^{n} a_{j}(t, x) D_{x_{j}}+b(t, x),
$$

where $a_{j}, b \in C\left([0, T] ; \mathcal{B}^{\infty}\left(\mathbb{R}^{n}\right)\right)$. Moreover, we suppose the condition

$$
\left|\Im a_{j}(t, x)\right| \leq \frac{C}{\langle x\rangle^{\sigma}} \text { with } \sigma \in(0,1), \quad(t, x) \in[0, T] \times \mathbb{R}^{n},
$$

where we use the notation $\langle\cdot\rangle^{2}=1+|\cdot|^{2}$. Mainly, we are looking for well-posedness of the Cauchy problem (1)-(3) in suitable spaces of functions of Gevrey regularity. We say that (1) is globally in time well-posed in the couple of spaces of functions (or distributions) $(X, Y)$ if for every choice of $g \in X$ there exists a unique solution $u \in C([0, T], Y)$ and for every $t \in[0, T]$ we have $\|u(t, \cdot)\|_{Y} \leq C_{t}\|g\|_{X}$ for a function $C_{t} \in C[0, T]$; we are going to say that (1) is locally in time well-posed in $(X, Y)$ if there exists $T^{*} \leq T$ such that there exists a unique solution $u \in C\left(\left[0, T^{*}\right], Y\right)$ and for every $t \in\left[0, T^{*}\right]$ we have $\|u(t, \cdot)\|_{Y} \leq C_{t}\|g\|_{X}$ for a function $C_{t} \in C\left[0, T^{*}\right]$.

It is well known, see [7], that the condition (3) allows to prove that if the coefficients of $S$ belong to the Gevrey space $G^{s_{0}}, s_{0}<\frac{1}{1-\sigma}$, then the Cauchy problem (1) is globally in time well-posed in Gevrey spaces $G^{s}$ for $s_{0} \leq s<\frac{1}{1-\sigma}$. In the critical case $s=\frac{1}{1-\sigma}$, one has local in time well-posedness of the Cauchy problem (1), only. The Cauchy problem (1) is not well-posed, neither in $H^{\infty}$ nor in $G^{s}$ for $s>\frac{1}{1-\sigma}$. Here, we refer to the necessity results from [4] and [3].

We recall that, given $s>1$, the Gevrey class $G^{s}\left(\mathbb{R}^{n}\right)$ consists of $C^{\infty}$ functions $f=f(x)$ such that

$$
\left|\partial_{x}^{\alpha} f(x)\right| \leq C A^{|\alpha|}|\alpha| !^{s} \text { for all } x \in \mathbb{R}^{n}, \alpha \in \mathbb{N}^{n}
$$

and with positive constants $A$ and $C$. Suitable subclasses of $G^{s}\left(\mathbb{R}^{n}\right)$ consist of functions $f \in L^{2}\left(\mathbb{R}^{n}\right)$ such that $\mathrm{e}^{\rho\langle D\rangle^{1 / s}} f \in L^{2}\left(\mathbb{R}^{n}\right)$ for some $\rho>0$. In [7] the authors show that if $g \in H^{m}$ is such that $\mathrm{e}^{\rho\langle D\rangle^{1 / s}} g \in H^{m}$ for some $m \in \mathbb{R}$ and $\rho>0$, then the Cauchy problem admits a unique solution $u$ such that $\mathrm{e}^{\tau\langle D\rangle^{1 / s}} u \in H^{m}$ at any $t \in[0, T]$ for a suitable $\tau=\tau(t) \leq \rho$. Since $\mathrm{e}^{\tau\langle D\rangle^{1 / s}}$ is a pseudo-differential operator of infinite order, the solution presents, with respect to the data, a loss of regularity, usually referred to as "loss of (an infinite number of) derivatives" in the mathematical literature.

The aim of the present paper is to give an answer to the following two questions:

Q1 Let us suppose that the data $g$ belongs to a weighted $H^{m}$ space with $m \geq 0$. Can we obtain at least a local (in time) Sobolev solution which is valued in an, in general, other weighted $H^{m}$ space? If yes, then the regularities of the solution and the data with respect to the spatial variables coincide. So, it turns out that the solution is valued in $H_{\mathrm{loc}}^{m}$ with respect to $x$.

Q2 What about well-posedness results in spaces with Gevrey regularity $G^{s}$ with $s>\frac{1}{1-\sigma}$ ?

As far as the authors know, the smoothing effect coming from decay of Cauchy data has been studied in the literature but not from the point of view of well-posedness, at least in question Q2. Some results concerning question Q1 are available under some stronger conditions with respect to (3). We describe hereafter briefly the state-of-the-art.

- In the particular case $a_{j} \equiv 0$ for $j=1, \ldots, n$, in [5] the author proved that if $g$ belongs to the weighted $L^{2}$ space with the weight $\langle x\rangle^{k}$, then there exists a uniquely determined 
Sobolev solution $u$ with a better regularity in $x$, but it belongs to a weighted Sobolev space with weight $\langle x\rangle^{-k}$ instead. More precisely,

$$
\langle x\rangle^{k} g \in H^{0} \text { with } k>0 \text { implies }\langle x\rangle^{-k} u(t, \cdot) \in H^{k} \text { for all } t>0 .
$$

The $H^{k}$ norm of $\langle x\rangle^{-k} u(t, \cdot)$ blows up as $t^{-k}$ for $t \rightarrow 0^{+}$. We have a smoothing effect but no well-posedness.

- In [6] the author considered the case $1 \leq s<\frac{1}{1-\sigma}$ and proved that, under assumption (3) and the additional decay assumption

$$
\left|\partial_{x}^{\alpha} a_{j}(t, x)\right| \leq C(\rho\langle x\rangle)^{-|\alpha|}|\alpha| !^{s},
$$

one has

$$
\begin{aligned}
& \mathrm{e}^{k\langle x\rangle^{1-\sigma}} g \in H^{0} \text { with } k>0 \text { implies } \\
& \quad\left|\partial_{x}^{\alpha} u(t, \cdot)\right| \leq C(\rho|t|)^{-|\alpha|} \alpha !^{s} \mathrm{e}^{c\langle x\rangle^{1-\sigma}} \text { for all } t>0
\end{aligned}
$$

with a suitable positive constant $c$. The Gevrey semi-norms of the classical solutions blow up as $t \rightarrow 0^{+}$. The smoothing effect is not due to any well-posedness result. We notice also that a decay behavior is assumed for all spatial derivatives of the coefficients.

- In [1] the authors proved that, in the framework of the SG calculus (so with coefficients $a_{j}, b$ possibly admitting an algebraic growth with respect to $x$ ) and if (3) holds with $\sigma=1$, the assumption that the data $g$ belongs to a Sobolev space with weight $\langle x\rangle^{k}$ gives a unique Sobolev solution with the same regularity as the data, but from another weighted space. More precisely, ${ }^{1}$

$$
\langle x\rangle^{k} g \in H^{m}, \quad m \geq 0, \text { implies }\langle x\rangle^{k-c} u(t, \cdot) \in H^{m} \text { for all } t \in[0, T]
$$

with a suitable $c>0$ with bounded norm with respect to $t \in[0, T]$. We recall that the $\mathrm{SG}$ (Symbol Global) calculus requires symbol like behavior of the coefficients also with respect to the spatial variables.

- A partial answer to question Q1 has been given, again in the framework of the SG calculus, as a by-product of [2]. Under the assumption

$$
a_{j}, b \in C\left([0, T], G^{s_{0}}\right), s_{0}<\frac{1}{1-\sigma} \text { and }\left|\partial_{x}^{\beta} \Im a_{j}(t, x)\right| \leq C^{|\beta|+1} \beta !^{s_{0}}\langle x\rangle^{-\sigma-|\beta|}
$$

we have

$$
\begin{gathered}
\mathrm{e}^{k\langle x\rangle^{1-\sigma}} g \in H^{m}, \quad m \geq 0, \quad k>0 \text { implies } \\
\mathrm{e}^{(k-c)\langle x\rangle^{\frac{1}{s}}} u(t, \cdot) \in H^{m} \text { for all } t \in[0, T]
\end{gathered}
$$

with a suitable $c>0$ and for every $s \in\left[s_{0}, \frac{1}{1-\sigma}\right.$ ) with bounded norm with respect to $t \in[0, T]$. This means that, the description of data from a weighted Sobolev space with a suitable exponential weight gives a uniquely determined Sobolev solution valued in the same Sobolev space but with either a slower increasing exponential weight (if $c<k$ ) or an exponentially decreasing weight (if $c>k$ ) as $|x| \rightarrow \infty$.

In the present paper, we are going to state and prove our main result, Theorem 1, which gives an answer to question Q2 and provides, as a corollary, the answer to question Q1, see Corollary 1 here below.

To state our main result we introduce the following function spaces.

1 We restrict ourselves to Sobolev solutions with respect to the spatial variables. For this reason we explain the result for $m \geq 0$. Several steps of our approach can be generalized to $m \in \mathbb{R}$, too. 
Definition 1 For given $m \geq 0, \sigma \in(0,1), s_{1}, s_{2} \in\left(\frac{1}{1-\sigma}, \infty\right], A>0, \rho>0$, we define

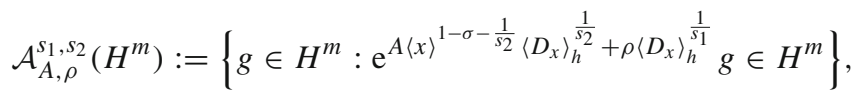

and the projective and inductive limit of these spaces, respectively, by

$$
\mathcal{A}_{s_{1}, s_{2}}\left(H^{m}\right)=\bigcap_{A, \rho>0} \mathcal{A}_{A, \rho}^{s_{1}, s_{2}}\left(H^{m}\right) \text { and } \mathcal{A}^{s_{1}, s_{2}}\left(H^{m}\right)=\bigcup_{A, \rho>0} \mathcal{A}_{A, \rho}^{s_{1}, s_{2}}\left(H^{m}\right) .
$$

Moreover, for given $s_{1}>1$ we define for every $s_{2} \in\left[s_{1}, \infty\right], A>0, \rho \geq 0$ the space

$$
\mathcal{B}_{A, \rho}^{s_{1}, s_{2}}\left(H^{m}\right):=\left\{g \in\left(\mathcal{A}_{s_{1}, s_{2}}\left(H^{m}\right)\right)^{*}: \mathrm{e}^{-A\langle x\rangle^{1-\sigma-\frac{1}{s_{2}}}\left\langle D_{x}\right\rangle_{h}^{\frac{1}{s_{2}}}+\rho\left\langle D_{x}\right\rangle_{h}^{\frac{1}{s_{1}}}} g \in H^{m}\right\} .
$$

Here, $\left(\mathcal{A}_{s_{1}, s_{2}}\left(H^{m}\right)\right)^{*}$ denotes the dual space to $\mathcal{A}_{s_{1}, s_{2}}\left(H^{m}\right)$. Finally, we define

$$
\mathcal{B}^{s_{1}, s_{2}}\left(H^{m}\right)=\bigcup_{A>0, \rho \geq 0} \mathcal{B}_{A, \rho}^{s_{1}, s_{2}}\left(H^{m}\right)
$$

Remark 1 Notice that in the limit case $\frac{1}{s_{1}}=\frac{1}{s_{2}}=0$ we get

$$
\mathcal{A}^{\infty, \infty}\left(H^{m}\right)=\left\{g \in H^{m}: \mathrm{e}^{A\langle x\rangle^{1-\sigma}} g \in H^{m} \text { for some } A>0\right\},
$$

a weighted space of $H^{m}$-functions with an exponentially increasing weight at infinity, and

$$
\mathcal{B}^{\infty, \infty}\left(H^{m}\right)=\left\{g \in H_{\mathrm{loc}}^{m}: \mathrm{e}^{-A\langle x\rangle^{1-\sigma}} g \in H^{m} \text { for some } A>0\right\},
$$

a weighted space of $H^{m}$-functions with an exponentially decaying weight at infinity.

We now present the main result of this paper, which gives an answer to question Q2.

Theorem 1 Assume that the data $g \in \mathcal{A}_{A, \rho}^{s_{1}, s_{2}}\left(H^{m}\right)$ for suitable $m \geq 0, \sigma \in(0,1), s_{1}, s_{2} \in$ $\left(\frac{1}{1-\sigma}, \infty\right], s_{2} \geq s_{1}$, and $A, \rho>0$. Then the Cauchy problem

$$
D_{t} u-\Delta u+\sum_{j=1}^{n} a_{j}(t, x) D_{x_{j}} u+b(t, x) u=0, u(0, x)=g(x),
$$

with $a_{j}, b \in C\left([0, T], G^{\frac{1}{1-\sigma}}\right)$, where the coefficients $a_{j}$ satisfy (3) for $j=1, \ldots, n$, admits a uniquely determined local (in time) Sobolev solution $u$ such that for every $t \in\left[0, T^{*}\right]$, $T^{*} \leq T$ small enough, we have

$$
u(t, \cdot) \in \bigcap_{\frac{1}{s} \in\left[0, \frac{1}{s_{1}}\right]} \mathcal{B}^{s_{1}, s}\left(H^{m}\right) .
$$

Moreover, for every $s \geq s_{1}$, there exists a function $C_{t}$ continuous on $\left[0, T^{*}\right]$ such that for every $t \in\left[0, T^{*}\right]$ the following energy estimate holds:

$$
\|u(t, \cdot)\|_{\mathcal{B}_{A, M(T-t)}^{s_{1}, s}\left(H^{m}\right)} \leq C_{t}\|g\|_{\mathcal{A}_{A, \rho}^{s_{1}, s_{2}}\left(H^{m}\right)} \cdot
$$

We remark that the estimate (6) gives local in time well-posedness of (1) in the couple of spaces $\left(\mathcal{A}^{s_{1}, s_{2}}, \mathcal{B}^{s_{1}, s}\right)$ for every $s \geq s_{1}, s_{2} \geq s_{1}$ and $s_{1}, s_{2} \in\left(\frac{1}{1-\sigma}, \infty\right]$.

If we choose in (4), (5) the parameters $s_{1}, s_{2}$ formally as $\frac{1}{s_{1}}=\frac{1}{s_{2}}=0$, then we obtain from Theorem 1 the following statement. 
Corollary 1 If the data $g \in H^{m}, m \geq 0$ is such that $\mathrm{e}^{A\langle x\rangle^{1-\sigma}} g \in H^{m}$ for a positive constant $A$, then the Cauchy problem

$$
D_{t} u-\Delta u+\sum_{j=1}^{n} a_{j}(t, x) D_{x_{j}} u+b(t, x) u=0, u(0, x)=g(x),
$$

with $a_{j}, b \in C\left([0, T], G^{\frac{1}{1-\sigma}}\right)$, where the coefficients $a_{j}$ satisfy (3) for $j=1, \ldots, n$, admits a uniquely determined local (in time) Sobolev solution $u$ such that for every $t \in\left[0, T^{*}\right]$, $T^{*} \leq T$ small enough, we have $\mathrm{e}^{-A^{\prime}\langle x\rangle^{1-\sigma}} u(t, \cdot) \in H^{m}$, where $A^{\prime}>0$ is a suitable constant. Consequently, $u(t, \cdot)$ belongs to $H_{\mathrm{loc}}^{m}\left(\mathbb{R}^{n}\right)$. Moreover, there exists a positive constant $M$ and a function $C_{t}$ continuous on $\left[0, T^{*}\right]$ such that for every $t \in\left[0, T^{*}\right]$

$$
\left\|\mathrm{e}^{(A-4 M)\langle x\rangle^{1-\sigma}} u(t, \cdot)\right\|_{H^{m}} \leq C_{t}\left\|\mathrm{e}^{A\langle x\rangle^{1-\sigma}} g\right\|_{H^{m}},
$$

i.e., the Cauchy problem is locally in time well-posed in weighted Sobolev spaces.

The result of Corollary 1, which is an answer to question Q1, implies that if the data $g$ belongs to a Sobolev space $H^{m}$ with an exponentially increasing weight, then the Sobolev solution is still valued in the same Sobolev space with an exponentially decreasing weight for $|x| \rightarrow \infty$.

Remark 2 We remark that, in comparison with [2] in the case of uniformly bounded in $x$ coefficients and in comparison with [6], we obtain by Corollary 1 a Sobolev solution valued in $H_{\mathrm{loc}}^{m}$ without any assumption on the spatial derivatives of $\Im a_{j}$. Furthermore, in comparison with [6], where a pointwise estimate for $u$ is given with a time-dependent constant tending to infinity for $t \rightarrow+0$, we have to mention that here, since we do not look for smoothing, we obtain for the solution $u$ an energy estimate on the whole interval $\left[0, T^{*}\right]$.

To conclude this section, we point out that in the particular case $s_{2}=\infty$ our main result reads as follows:

Corollary 2 Assume that the data $g \in H^{m}, m \geq 0$, is such that

$$
\mathrm{e}^{A\langle x\rangle^{1-\sigma}+\rho\left\langle D_{x}\right\rangle_{h}^{\frac{1}{s_{1}}}} g \in H^{m}
$$

for given $\sigma \in(0,1), s_{1} \in\left(\frac{1}{1-\sigma}, \infty\right], A, \rho>0$. Then, the Cauchy problem

$$
D_{t} u-\Delta u+\sum_{j=1}^{n} a_{j}(t, x) D_{x_{j}} u+b(t, x) u=0, u(0, x)=g(x),
$$

with $a_{j}, b \in C\left([0, T], G^{\frac{1}{1-\sigma}}\right), a_{j}$ satisfying (3) for $j=1, \ldots, n$, admits a uniquely determined local (in time) Sobolev solution $u$ such that for every $t \in\left[0, T^{*}\right], T^{*} \leq T$ small enough, we have that

$$
u(t, \cdot) \in \bigcap_{\frac{1}{s} \in\left[0, \frac{1}{s_{1}}\right]} \mathcal{B}^{s_{1}, s}\left(H^{m}\right) .
$$

In particular, taking $s=\infty$ we get

$$
\mathrm{e}^{-A\langle x\rangle^{1-\sigma}+\rho^{\prime}\left\langle D_{x}\right\rangle_{h}^{\frac{1}{s_{1}}}} u(t, \cdot) \in H^{m}
$$


for a suitable positive $\rho^{\prime}$. Moreover, there exists a function $C_{t}$ continuous on $\left[0, T^{*}\right]$ such that for every $t \in\left[0, T^{*}\right]$ the following estimate holds:

$$
\left\|\mathrm{e}^{-A\langle x\rangle^{1-\sigma}+\rho^{\prime}\left\langle D_{x}\right\rangle_{h}^{\frac{1}{s_{1}}}} u(t, \cdot)\right\|_{H^{m}} \leq C_{t}\left\|\mathrm{e}^{A\langle x\rangle^{1-\sigma}+\rho\left\langle D_{x}\right\rangle_{h}^{\frac{1}{s_{1}}}} g\right\|_{H^{m}} .
$$

Remark 3 We remark that Corollary 2 states that if we start with a data having Gevrey-type regularity of exponent $s_{1}$ and belonging to a weighted space with exponentially increasing weight $\mathrm{e}^{A\langle x\rangle^{1-\sigma}}$, we find a unique solution with the same Gevrey regularity belonging to a weighted space with exponentially decreasing weight $\mathrm{e}^{-A\langle x\rangle^{1-\sigma}}$ for every $s_{1}>1 /(1-\sigma)$. This result is consistent with the one obtained in [2] for the critical case $s=\frac{1}{1-\sigma}$. We can so overcome the critical index $1 /(1-\sigma)$ for $G^{s}$ well- posedness by allowing a suitable loss of asymptotic behavior as $|x| \rightarrow \infty$ in the used weights.

Remark 4 We believe that this loss of asymptotic behavior is sharp in the sense that a smaller loss of asymptotic behavior may lead to a non- well-posed Cauchy problem in suitable Gevrey classes. Indeed, in a forthcoming paper, we aim to construct a Cauchy data $g \in H^{m}$ such that

$$
\mathrm{e}^{A\langle x\rangle^{1-\alpha}+\rho\langle D\rangle_{h}^{\frac{1}{s_{1}}}} g \in H^{m} \text { for some } \sigma<\alpha<1
$$

but for every $s_{1} \in\left(\frac{1}{1-\sigma}, \frac{1}{1-\alpha}\right]$ we have $\mathrm{e}^{-A^{\prime}\langle x\rangle^{1-\alpha}+\rho^{\prime}\langle D\rangle_{h}^{\frac{1}{s_{1}}}} u(t, \cdot) \notin H^{m}$.

A result of this type would confirm that the "extreme" loss of behavior (from the weight $\mathrm{e}^{A\langle x\rangle^{1-\sigma}}$ to the weight $\mathrm{e}^{A\langle x\rangle^{1-\sigma}}$ ) that we observe in Corollary 2 (and, of course, in Theorem 1 ) is necessary to gain, by assuming a decay on the data, well-posedness in $G^{s}$ also for $s>1 /(1-\sigma)$.

The strategy of the proof of Theorem 1 (and, with minor changes, of the two corollaries) is as follows:

- We perform the change of variable

$$
v(t, x)=\mathrm{e}^{\Lambda}(t, x, D) u(t, x),
$$

where $\mathrm{e}^{\Lambda}=\mathrm{op}\left(\mathrm{e}^{\Lambda(t, x, \xi)}\right)$ is a pseudo-differential operator of infinite order with symbol $\mathrm{e}^{\Lambda(t, x, \xi)}$, constructed in a way such that the Cauchy problem $S_{\Lambda} v=0, v(0)=g_{\Lambda}$ is equivalent to (1). It has data $g_{\Lambda} \in H^{m}$ and $S_{\Lambda}$ has the structure

$$
\begin{aligned}
S_{\Lambda}= & D_{t}-\triangle_{x}+\sum_{j=1}^{n}\left\{a_{j}(t, x) D_{x_{j}}+2 i \mathrm{op}\left(\left(\partial_{x_{j}} \Lambda\right) \xi_{j}\right)\right\} \\
& \quad+i \mathrm{op}\left(\partial_{t} \Lambda\right)+r_{1-\sigma}(t, x, D)+r_{0}(t, x, D) \\
= & : D_{t}-\triangle_{x}-i A_{\Lambda}(t, x, D),
\end{aligned}
$$

where $r_{1-\sigma}$ and $r_{0}$ are pseudo-differential operators of order $1-\sigma$ and $r_{0}$, respectively.

- By a correct choice of $\Lambda$, while writing an energy estimate for $v$ it is possible to use the contribution coming from $\sum_{j=1}^{n} 2 i o p\left(\left(\partial_{x_{j}} \Lambda\right) \xi_{j}\right)$ to compensate the contribution coming from $\sum_{j=1}^{n} a_{j}(t, x) D_{x_{j}}$ and to use the contribution coming from $i o p\left(\partial_{t} \Lambda\right)$ to compensate the contribution coming from $r_{1-\sigma}$, obtaining that

$$
2 \Re\left\langle A_{\Lambda}(t, x, D) v, v\right\rangle \geq 0,
$$

that is, the Cauchy problem for $v$ is well-posed in Sobolev spaces. 
- The inverse change of variable $u=\left(\mathrm{e}^{\Lambda}\right)^{-1} v$ gives the solution to the original Cauchy problem.

The construction of the correct function $\Lambda$ is the crux of the matter, and it is quite technical. Indeed, several features are required for $\Lambda$ and the transformation needs obviously to be invertible. The symbol $\Lambda$ will be of the form

$$
\Lambda(t, x, \xi)=\tilde{\Lambda}(t, x, \xi)+M(T-t)\langle\xi\rangle_{h}^{\frac{1}{s_{1}}}
$$

with $M>0$ large, to be chosen at the end of the proof to get (9), where the second term in (10) rules the Gevrey regularity of the solution and the first one, which rules the behavior at infinity, is constructed in such a way that $\mathrm{e}^{\tilde{\Lambda}}$ is invertible (for $h \geq 1$ large enough and $T \leq T^{*}$ small enough). Moreover, it satisfies the crucial inequality

$$
\partial_{t} \Lambda(t, x, \xi)+2 \sum_{j=1}^{n} \xi_{j} \partial_{x_{j}} \Lambda(t, x, \xi) \leq-M\langle x\rangle^{-\sigma}\langle\xi\rangle_{h} .
$$

This inequality will allow us to use the new terms appearing in $S_{\Lambda}$ for the compensation procedure described above. Notice that the restriction to a subinterval $\left[0, T^{*}\right]$ is needed for the invertibility of $\mathrm{e}^{\tilde{\Lambda}}$. Finally, the symbol $\tilde{\Lambda}$ that we construct has a special behavior of type $\langle x\rangle^{1-\sigma-\frac{1}{s}}\langle\xi\rangle_{h}^{\frac{1}{s}}$ for every $0 \leq \frac{1}{s} \leq 1-\sigma$. This particular behavior is very useful in the proof of our theorems; on one hand we can think that $\tilde{\Lambda}$ behaves like $\langle\xi\rangle_{h}^{1-\sigma}$ when we perform the change of variable (so we can apply the well-established theory for symbols uniformly bounded in space to compute the conjugation $\left.\mathrm{e}^{\tilde{\Lambda}} S_{\Lambda}\left(\mathrm{e}^{\tilde{\Lambda}}\right)^{-1}\right)$, and on the other hand we can think that $\tilde{\Lambda}$ behaves like $\langle x\rangle^{1-\sigma}$ when we recapture $u=\mathrm{e}^{-M(T-t)\left\langle D_{x}\right\rangle_{h}^{1 / s_{1}}}\left(\mathrm{e}^{\tilde{\Lambda}}\right)^{-1} v$, obtaining a solution $v$ with no loss of regularity, but possibly different behavior as $|x| \rightarrow \infty$.

The paper is structured as follows:

- In Sect. 2, we present a class of symbols with Gevrey regularity, the corresponding class of pseudo-differential operators, and the class of Gevrey-Sobolev spaces, where these operators act continuously in suitable scales of spaces. Moreover, we state the invertibility of operators of infinite order of the form $\mathrm{e}^{\Lambda}$ and we describe the structure of the conjugation $\mathrm{e}^{\Lambda} S\left(\mathrm{e}^{\Lambda}\right)^{-1}$.

- In Sect. 3, we perform the change of variable, constructing $\Lambda$, checking its invertibility and deriving explicitly the equivalent Cauchy problem.

- Section 4 is devoted to a crucial result, Lemma 1, which states the continuity of the maps $\mathrm{e}^{\Lambda}: \mathcal{A}^{s_{1}, s_{2}} \longrightarrow H^{m}$ and $\left(\mathrm{e}^{\Lambda}\right)^{-1}: H^{m} \longrightarrow \mathcal{B}^{s_{1}, s}$. Moreover, we give the proof of the main theorem and of the corollaries. The continuity of $\mathrm{e}^{\Lambda}$ will allow us to study the Cauchy problem for $v$ in Sobolev spaces, the continuity of $\left(\mathrm{e}^{\Lambda}\right)^{-1}$ will provide the space of well-posedness for the original Cauchy problem.

A discussion about the characterization of $\mathcal{A}^{s_{1}, s_{2}}$ and $\mathcal{B}^{s_{1}, s}$ spaces via Fourier transform concludes the paper.

\section{Preliminaries}

In what follows, we are going to consider for $m \in \mathbb{R}$ and $s \geq 1$ symbols of Gevrey regularity in the following sense: we say that a given $C^{\infty}\left(\mathbb{R}^{2 n}\right)$ function $a=a(x, \xi)$ belongs to $S_{s}^{m}\left(\mathbb{R}^{n}\right)$ if it satisfies 


$$
\left|\partial_{\xi}^{\alpha} \partial_{x}^{\beta} a(x, \xi)\right| \leq C_{h} A^{|\alpha|+|\beta|}|\alpha+\beta| !^{s}\langle\xi\rangle_{h}^{m-|\alpha|}, \quad(x, \xi) \in \mathbb{R}^{2 n}, \alpha, \beta \in \mathbb{Z}_{+}^{n},
$$

for some constants $h>0, C_{h}>0$ and $A>1$. Here and in the following, we use the notation $\langle\xi\rangle_{h}^{2}:=h^{2}+|\xi|^{2}$. The space $S_{s}^{m}\left(\mathbb{R}^{n}\right)$ is a limit space in the following sense:

$$
S_{s}^{m}\left(\mathbb{R}^{n}\right):=\lim _{\ell \rightarrow+\infty} S_{s, \ell}^{m}\left(\mathbb{R}^{n}\right) \quad \text { with } \quad S_{s, \ell}^{m}\left(\mathbb{R}^{n}\right):=\lim _{\substack{\rightarrow \rightarrow+\infty \\ s, \ell, A}} S_{s}^{m}\left(\mathbb{R}^{n}\right) .
$$

Here, $S_{s, A, \ell}^{m}\left(\mathbb{R}^{n}\right)$ denotes the Banach space of all symbols satisfying the conditions such that

$$
|a|_{m, s, A, \ell}:=\sup _{|\alpha+\beta| \leq \ell} \sup _{x, \xi}\left|\partial_{\xi}^{\alpha} \partial_{x}^{\beta} a(x, \xi)\right| A^{-|\alpha|-|\beta|}(|\alpha|+|\beta|) !^{-s}\langle\xi\rangle_{h}^{-m+|\alpha|}<+\infty .
$$

We are going to use pseudo-differential operators $p(x, D)=\mathrm{op}(p(x, \xi))$ with symbols $\sigma(p(x, D))=p(x, \xi) \in S_{s}^{m}\left(\mathbb{R}^{n}\right)$. These operators act continuously on the so-called Sobolev-Gevrey spaces, defined for $m \in \mathbb{R}, \rho>0, s \geq 1$ as follows:

$$
H_{\rho, s}^{m}\left(\mathbb{R}^{n}\right):=\left\{u \in \mathcal{S}^{\prime}\left(\mathbb{R}^{n}\right):\|u\|_{m, \rho, s}:=\left\|\mathrm{e}^{\rho\left\langle D_{x}\right\rangle^{\frac{1}{s}}} u\right\|_{H^{m}}<\infty\right\} .
$$

We are also going to deal with pseudo-differential operators of infinite order $\mathrm{e}^{\Lambda(x, D)}$ with symbols of the form $\mathrm{e}^{\Lambda(x, \xi)}$, where $\Lambda$ satisfies

$$
\left|\partial_{\xi}^{\alpha} \partial_{x}^{\beta} \Lambda(x, \xi)\right| \leq C_{\Lambda} A^{|\alpha|+|\beta|}|\alpha+\beta| !^{!}\langle\xi\rangle_{h}^{\frac{1}{s}-|\alpha|}, \quad(x, \xi) \in \mathbb{R}^{n}, \alpha, \beta \in \mathbb{Z}_{+}^{n}
$$

for a constant $C_{\Lambda}$ independent of the parameter $h \geq 1$ and $s>1$. By Theorem 6.14 in [8], operators of this form turn out to be invertible on $L^{2}$ by Neumann series for $h$ large enough and $C_{\Lambda}$ small enough. Indeed, let us consider the pseudo-differential operator $\mathrm{e}^{\Lambda(x, D)}$ with symbol $\mathrm{e}^{\Lambda(x, \xi)}$, and define its so-called reversed operator

$$
\left({ }^{R} \mathrm{e}^{\Lambda}\right)(x, D) u(x):=(2 \pi)^{-n} \int_{\mathbb{R}^{n}}\left(\int_{\mathbb{R}^{n}} \mathrm{e}^{i(x+y) \cdot \xi+\Lambda(y, \xi)} u(y) \mathrm{d} y\right) d \xi
$$

defined as an oscillatory integral. Then, we have the following properties:

1. $\mathrm{e}^{\Lambda}: H_{\rho, s}^{0}\left(\mathbb{R}^{n}\right) \longrightarrow H_{\rho-\rho^{\prime}, s}^{0}\left(\mathbb{R}^{n}\right)$ is a continuous mapping for $\left|\rho-\rho^{\prime}\right|<\delta A^{-\frac{1}{s}}$ and $\rho^{\prime}>C_{\Lambda}$,

2. ${ }^{R} \mathrm{e}^{\Lambda}: H_{\rho, s}^{0}\left(\mathbb{R}^{n}\right) \longrightarrow H_{\rho-\rho^{\prime}, s}^{0}\left(\mathbb{R}^{n}\right)$ is a continuous mapping for $|\rho|<\delta A^{-\frac{1}{s}}$ and $\rho^{\prime}>C_{\Lambda}$, where $\delta>0$ is a suitable constant, see [8, Part I, Proposition 6.7],

3. if we form the composition $\mathrm{e}^{\Lambda}\left({ }^{R} \mathrm{e}^{-\Lambda}\right)$, then we get

$$
\mathrm{e}^{\Lambda}\left({ }^{R} \mathrm{e}^{-\Lambda}\right)=I+r\left(x, D_{x}\right)
$$

where $r(x, \xi)$ has the asymptotic expansion

$$
r(x, \xi) \sim \sum_{j \geq 1} r_{j}(x, \xi), \quad r_{j}(x, \xi)=\sum_{|\alpha|=j} \frac{1}{\alpha !} \partial_{\xi}^{\alpha}\left(\mathrm{e}^{\Lambda(x, \xi)} D_{x}^{\alpha} \mathrm{e}^{-\Lambda(x, \xi)}\right)
$$

and satisfies

$$
\left|r_{(\beta)}^{(\alpha)}(x, \xi)\right| \leq C_{\alpha, \beta}\langle\xi\rangle_{h}^{\frac{1}{s}-1-\alpha} \leq C_{\alpha, \beta} h^{\frac{1}{s}-1}\langle\xi\rangle_{h}^{-\alpha},
$$

with $C_{\alpha, \beta}$ independent of $h$. 
Using these properties we can fix a large $h$ in order to have a bounded operator

$$
r\left(x, D_{x}\right): u \in H^{\mu} \rightarrow r\left(x, D_{x}\right) u \in H^{\mu} \text { with norm }\left\|r\left(x, D_{x}\right)\right\|_{H^{\mu} \rightarrow H^{\mu}}<1 .
$$

The operator $I+r\left(x, D_{x}\right)$ is invertible by Neumann series and its inverse operator is given by

$$
I+p\left(x, D_{x}\right), \quad p=\sum_{j=1}^{\infty}(-r)^{j} .
$$

This proves that the operator ${ }^{R} \mathrm{e}^{-\Lambda}(I+p)$ is the right inverse of $\mathrm{e}^{\Lambda}$. By similar arguments one proves the existence of a left inverse. Thus, the operator $\mathrm{e}^{\Lambda}$ is invertible, and the inverse operator is given by

$$
\left(\mathrm{e}^{\Lambda}\right)^{-1}=\left({ }^{R} \mathrm{e}^{-\Lambda}\right)(I+p) .
$$

Moreover, let us notice that the inverse has the structure

$$
\begin{aligned}
\left(\mathrm{e}^{\Lambda}\right)^{-1} & =\left({ }^{R} \mathrm{e}^{-\Lambda}\right)(I-r+\text { lower order terms }) \\
& =\left({ }^{R} \mathrm{e}^{-\Lambda}\right)\left(I-r_{1}+\text { lower order terms }\right) \\
& =\left({ }^{R} \mathrm{e}^{-\Lambda}\right) \operatorname{op}\left(1+\sum_{j=1}^{n} \partial_{\xi_{j}} D_{x_{j}} \Lambda(x, \xi)+\text { lower order terms }\right) .
\end{aligned}
$$

Remark 5 We may apply the same arguments to the operator $\mathrm{e}^{\Lambda^{\prime}}$ with symbol $\sigma\left(\Lambda^{\prime}\right)$ given by

$$
\sigma\left(\Lambda^{\prime}\right)(x, \xi)=A\langle x\rangle^{1-\sigma-\frac{1}{s_{2}}}\langle\xi\rangle_{h}^{\frac{1}{s_{2}}}+\rho\langle\xi\rangle_{h}^{\frac{1}{s_{1}}}
$$

In particular, we have

$$
\mathcal{A}_{A, \rho}^{s_{1}, s_{2}}\left(H^{m}\right)=\left\{u={ }^{R} \mathrm{e}^{-\Lambda^{\prime}} v: v \in H^{m}\right\} .
$$

Finally, Theorem 6.14 in [8] states that there exist $\delta>0$ and $h_{0}>1$ such that for every $h \geq h_{0}$ and $C_{\Lambda}<\delta A^{-\frac{1}{s}}$ the conjugation $\mathrm{e}^{\Lambda} p\left(\mathrm{e}^{\Lambda}\right)^{-1}$ makes sense for every operator $p(x, D)$ having the symbol $p(x, \xi) \in S_{s}^{m}\left(\mathbb{R}^{2 n}\right)$. Moreover, the conjugation has the following structure:

$$
\mathrm{e}^{\Lambda}(x, D) p(x, D)\left(\mathrm{e}^{\Lambda}(x, D)\right)^{-1}=p(x, D)+q(x, D)+r(x, D),
$$

where $r(x, \xi) \in S_{s}^{m-2\left(1-\frac{1}{s}\right)}\left(\mathbb{R}^{n}\right)$ and

$$
q(x, \xi)=\sum_{|\alpha|=1} \partial_{\xi}^{\alpha} p(x, \xi)\left(i \partial_{x}\right)^{\alpha} \Lambda(x, \xi)+\sum_{|\beta|=1} D_{x}^{\beta} p(x, \xi) \partial_{\xi}^{\beta} \Lambda(x, \xi) .
$$

\section{Change of variables}

To prove Theorem 1 and Corollary 1, we perform the change of variables

$$
v(t, x)=\mathrm{e}^{\Lambda(t, x, D)} u(t, x),
$$

by choosing a suitable symbol $\Lambda=\Lambda(t, x, \xi)$ with the following features: 
- the function $\Lambda$ has the form $\Lambda(t, x, \xi)=\tilde{\Lambda}(t, x, \xi)+\Lambda_{3}(t, \xi)$, where $\tilde{\Lambda}$ satisfies for an arbitrary $\mu>1$ the following symbol like estimates:

$$
\begin{aligned}
& \left|\partial_{\xi}^{\alpha} \partial_{x}^{\beta} \tilde{\Lambda}(t, x, \xi)\right| \leq C^{\alpha+\beta+1}|\alpha+\beta| !^{\mu}\langle x\rangle^{\delta-|\beta|}\langle\xi\rangle_{h}^{d-|\alpha|} \\
& \quad \text { for all } \delta, d \text { with } d \geq 0, \quad \delta+d=1-\sigma,
\end{aligned}
$$

where $C=C_{T}$ is a suitable positive constant which depends continuously on $T$ but which is independent of $h \geq 1$;

- $\Lambda_{3} \in S^{\frac{1}{s_{1}}}\left(\mathbb{R}^{n}\right)$ (recall that $\left.\frac{1}{s_{1}}<1-\sigma\right)$;

- the operator $\mathrm{e}^{\Lambda}$ is invertible for $h>0$ large enough;

- the operators of infinite order

$$
\mathrm{e}^{\Lambda}(t, x, D): \mathcal{A}_{A, \rho}^{s_{1}, s_{2}}\left(H^{m}\right) \longrightarrow H^{m} \text { and }{ }^{R} \mathrm{e}^{-\Lambda}(t, x, D): H^{m} \longrightarrow \mathcal{B}_{A, \rho}^{s_{1}, s_{2}}\left(H^{m}\right)
$$

are continuous mappings for suitable (large enough) $A$ and $\rho$, see Lemma 1 below;

- for sufficiently large constants $h>0$ and $M>0$ the following crucial inequality holds:

$$
\partial_{t} \Lambda(t, x, \xi)+2 \sum_{j=1}^{n} \xi_{j} \partial_{x_{j}} \Lambda(t, x, \xi) \leq-M\langle x\rangle^{-\sigma}\langle\xi\rangle_{h} .
$$

By this change of variable, choosing suitably the phase function $\Lambda$, we reduce the Cauchy problem

$$
S u=0, u(0, x)=g,
$$

to the equivalent Cauchy problem

$$
\begin{cases}S_{\Lambda} v=0, & S_{\Lambda}=D_{t}-\Delta_{x}-i A_{\Lambda}(t, x, D)+r_{0}(t, x, D), \\ v(0, x)=g_{\Lambda}, & g_{\Lambda}=\mathrm{e}^{\Lambda(0)} g,\end{cases}
$$

where the pseudo-differential operator $A_{\Lambda}$ satisfies the condition

$$
2 \Re\left\langle A_{\Lambda}(t, x, D) v, v\right\rangle \geq 0 .
$$

The remainder $r_{0}$ is a pseudo-differential operator of order zero. It turns out that this Cauchy problem is $L^{2}$ well-posed, and trivially also $H^{m}$ well-posed. Then, coming back to the original Cauchy problem, from $v \in C\left([0, T] ; H^{m}\right)$, using the structure of $\Lambda_{3}$ and (18) with $\delta=1-\sigma-\frac{1}{s}$ and $d=\frac{1}{s}, 0 \leq \frac{1}{s} \leq \frac{1}{s_{1}}$, we obtain that $u=\left(\mathrm{e}^{\Lambda}\right)^{-1} v$ satisfies for every $t \in\left[0, T^{*}\right]$ the condition

$$
\mathrm{e}^{-A\langle x\rangle^{1-\sigma-\frac{1}{s}}\langle D\rangle_{h}^{\frac{1}{s}}+\rho^{\prime}\langle D\rangle_{h}^{\frac{1}{s_{1}}}} u(t, \cdot) \in H^{m}
$$

with a suitable positive $\rho^{\prime}$. For this reason, $u(t, \cdot) \in \mathcal{B}_{A, \rho^{\prime}}^{s_{1}, s}\left(H^{m}\right)$ for $t \in\left[0, T^{*}\right]$ and every $0 \leq \frac{1}{s} \leq \frac{1}{s_{1}}$. More details are provided in the proofs of Theorem 1 and of Corollary 1 .

Remark 6 In the case of Corollary 1 , we take $\Lambda_{3}(t, \xi) \equiv 0$. By choosing $\delta=1-\sigma$ and $d=0$, we arrive at a result without any loss of regularity. The other limit case $\delta=0$, $d=1-\sigma$, corresponds to a result of [7].

We choose

$$
\Lambda(t, x, \xi):=\tilde{\Lambda}(t, x, \xi)+\Lambda_{3}(t, \xi), \quad \tilde{\Lambda}(t, x, \xi):=\Lambda_{1}(t, x, \xi)+\Lambda_{2}(x, \xi),
$$


where

$$
\begin{aligned}
\Lambda_{1}(t, x, \xi) & :=M(T-t)\langle x\rangle^{-\sigma}\langle\xi\rangle_{h}\left(1-\chi\left(\frac{\langle x\rangle}{\epsilon\langle\xi\rangle_{h}}\right)\right), \\
\Lambda_{2}(x, \xi) & :=\chi\left(\frac{2\langle x\rangle}{\epsilon\langle\xi\rangle_{h}}\right) \lambda(x, \xi), \\
\Lambda_{3}(t, \xi) & :=M(T-t)\langle\xi\rangle_{h}^{\frac{1}{s_{1}}},
\end{aligned}
$$

under the following assumptions:

- $M$ is a sufficiently large positive constant to be chosen later on;

$-\epsilon>0$ is an arbitrarily small constant depending on $M$;

- $h \geq 1$ will be chosen later on, in fact, we will choose $h \geq h_{0}$ with $h_{0}>0$ large enough to have the invertibility of $\mathrm{e}^{\tilde{\Lambda}}$;

- $\chi \in C_{0}^{\infty}(\mathbb{R})$ is such that $0 \leq \chi(t) \leq 1, t \chi^{\prime}(t) \leq 0$ for all $t \in \mathbb{R}, \chi(t)=1$ for $|t| \leq \frac{1}{2}$, $\chi(t)=0$ for $|t| \geq 1$, and $\left|\chi^{(k)}(t)\right| \leq A_{0}^{k+1} k !^{\mu}$ for some $\mu>1$ to be chosen later on;

$-\lambda=\lambda(x, \xi)$ is a solution to the inequality

$$
\sum_{j=1}^{n} \xi_{j} \partial_{x_{j}} \lambda(x, \xi) \leq-M\langle x\rangle^{-\sigma}\langle\xi\rangle_{h},
$$

with a large constant $M$ to be chosen later on.

The function $\lambda$ is given as follows:

$$
\lambda(x, \xi):=-M\left(\lambda_{1}(x, \xi) \chi\left(\frac{2 x \cdot \omega}{\langle x\rangle}\right)-\lambda_{2}(x, \xi)\left(1-\chi\left(\frac{2 x \cdot \omega}{\langle x\rangle}\right)\right)\right)
$$

with $\omega=\xi /|\xi|$, where

$$
\lambda_{1}(x, \xi):=\int_{0}^{x \cdot \omega}\langle x-\tau \omega\rangle_{h}^{-\sigma} \mathrm{d} \tau \text { and } \lambda_{2}(x, \xi):=\int_{0}^{x \cdot \omega}\langle\tau\rangle_{h}^{-\sigma} \mathrm{d} \tau .
$$

We know by Lemma 4 of [2] that there exists a constant $C_{\sigma}$ independent of $h$ and $M$ such that the function $\lambda=\lambda(x, \xi)$ which is defined in (27) satisfies the following estimate for every $\alpha, \beta \in \mathbb{Z}_{+}^{n}$ :

$$
\left|\partial_{\xi}^{\alpha} \partial_{x}^{\beta} \lambda(x, \xi)\right| \leq M C_{\sigma}^{|\alpha|+|\beta|+1}|\alpha+\beta|^{\mu}\langle x\rangle^{1-\sigma-|\beta|}|\xi|^{-|\alpha|}
$$

for every $(x, \xi) \in \mathbb{R}^{2 n}$ with $|\xi|>1$. Notice that it is enough to estimate $\lambda=\lambda(t, x, \xi)$ for $|\xi|>1$ because $\Lambda_{2}$ is supported in the region

$$
\langle\xi\rangle_{h} \geq \frac{2\langle x\rangle}{\epsilon} \geq \frac{2}{\epsilon}>\langle 1\rangle_{h}
$$

if $\epsilon$ is small enough, thanks to the use of the cut-off function $\chi$.

Now, since in (24) the term $\Lambda_{2}=\Lambda_{2}(x, \xi)$ is given by $\chi\left(\frac{2\langle x\rangle}{\epsilon\langle\xi\rangle_{h}}\right) \lambda(x, \xi)$ and due to (see [7], formula (2.6))

$$
\left|\partial_{\xi}^{\alpha} \partial_{x}^{\beta}\left(\chi\left(\frac{2\langle x\rangle}{\epsilon\langle\xi\rangle_{h}}\right)\right)\right| \leq C_{1} A_{1}^{|\alpha+\beta|}|\alpha+\beta|^{\mu}\langle x\rangle^{-|\beta|}\langle\xi\rangle_{h}^{-|\alpha|}
$$


with $C_{1}, A_{1}$ independent of $\epsilon$, it follows that for every $\delta \in[-\sigma, 1-\sigma], d \in[0,1]$ satisfying $\delta+d=1-\sigma$ we have the relations

$$
\begin{aligned}
\partial_{\xi}^{\alpha} \partial_{x}^{\beta} \Lambda_{2}(x, \xi) & =\sum_{\alpha_{1}+\alpha_{2}=\alpha} \sum_{\beta_{1}+\beta_{2}=\beta}\left(\begin{array}{c}
\alpha \\
\alpha_{1}
\end{array}\right)\left(\begin{array}{c}
\beta \\
\beta_{1}
\end{array}\right) \partial_{\xi}^{\alpha_{1}} \partial_{x}^{\beta_{1}} \chi\left(\frac{2\langle x\rangle}{\epsilon\langle\xi\rangle_{h}}\right) \partial_{\xi}^{\alpha_{2}} \partial_{x}^{\beta_{2}} \lambda(x, \xi), \\
\left|\partial_{\xi}^{\alpha} \partial_{x}^{\beta} \Lambda_{2}(x, \xi)\right| & \leq M C_{\sigma}^{|\alpha|+|\beta|+1}|\alpha+\beta| !^{\mu}\langle x\rangle^{1-\sigma-|\beta|}\langle\xi\rangle_{h}^{-|\alpha|} \\
& =M C_{\sigma}^{|\alpha|+|\beta|+1}|\alpha+\beta|^{\mu}\langle x\rangle^{\delta-|\beta|}\langle x\rangle^{d}\langle\xi\rangle_{h}^{-|\alpha|} \\
& \leq M\left(\frac{\epsilon}{2}\right)^{d} \tilde{C}_{\sigma}^{|\alpha|+|\beta|+1}|\alpha+\beta|^{\mu}\langle x\rangle^{\delta-|\beta|}\langle\xi\rangle_{h}^{d-|\alpha|}
\end{aligned}
$$

with a constant $C_{\sigma}$ which is independent of $h, M, \epsilon$. Here, we use the inequality $\langle x\rangle \leq \frac{\epsilon}{2}\langle\xi\rangle_{h}$ on the support of $\chi\left(\frac{\langle x\rangle}{\epsilon\langle\xi\rangle_{h}}\right)$. As it concerns the term $\Lambda_{1}=\Lambda_{1}(t, x, \xi)$ in (22), we have

$$
\begin{aligned}
\partial_{\xi}^{\alpha} \partial_{x}^{\beta} \Lambda_{1}(t, x, \xi)= & M(T-t) \sum_{\alpha_{1}+\alpha_{2}=\alpha} \sum_{\beta_{1}+\beta_{2}=\beta}\left(\begin{array}{c}
\alpha \\
\alpha_{1}
\end{array}\right)\left(\begin{array}{c}
\beta \\
\beta_{1}
\end{array}\right) \partial_{\xi}^{\alpha_{1}}\langle\xi\rangle_{h} \partial_{x}^{\beta_{1}}\langle x\rangle^{-\sigma} \\
& \times \partial_{\xi}^{\alpha_{2}} \partial_{x}^{\beta_{2}}\left(1-\chi\left(\frac{\langle x\rangle}{\epsilon\langle\xi\rangle_{h}}\right)\right), \\
\left|\partial_{\xi}^{\alpha} \partial_{x}^{\beta} \Lambda_{1}(t, x, \xi)\right| \leq & M(T-t) C_{2}^{|\alpha|+|\beta|+1}|\alpha+\beta| !^{\mu}\langle x\rangle^{-\sigma-|\beta|}\langle\xi\rangle_{h}^{1-|\alpha|} \\
= & M(T-t) C_{2}^{|\alpha|+|\beta|+1}|\alpha+\beta| !^{\mu}\langle x\rangle^{\delta-|\beta|}\langle x\rangle^{-\sigma-\delta}\langle\xi\rangle_{h}^{d-|\alpha|}\langle\xi\rangle_{h}^{1-d} \\
\leq & M(T-t)\left(\frac{2}{\epsilon}\right)^{1-d} C_{2}^{|\alpha|+|\beta|+1}|\alpha+\beta|^{\mu}\langle x\rangle^{\delta-|\beta|}\langle\xi\rangle_{h}^{d-|\alpha|}\langle x\rangle^{1-d-\sigma-\delta} \\
= & M(T-t)\left(\frac{2}{\epsilon}\right)^{1-d} C_{2}^{|\alpha|+|\beta|+1}|\alpha+\beta| !^{\mu}\langle x\rangle^{\delta-|\beta|}\langle\xi\rangle_{h}^{d-|\alpha|},
\end{aligned}
$$

for every $\delta \in[-\sigma, 1-\sigma], d \in[0,1]$ satisfying $\delta+d=1-\sigma$, with a constant $C_{2}$ which is independent of $h, M, T, \epsilon$. Here, we use the inequality $\langle\xi\rangle_{h} \leq \frac{2}{\epsilon}\langle x\rangle$ on the support of $1-\chi\left(\frac{\langle x\rangle}{\epsilon\langle\xi\rangle_{h}}\right)$. Summing up we arrive for $\delta+d=1-\sigma$ with $\delta \in[-\sigma, 1-\sigma], d \in[0,1]$ at the following estimate:

$$
\left|\partial_{\xi}^{\alpha} \partial_{x}^{\beta} \tilde{\Lambda}(t, x, \xi)\right| \leq M\left(T\left(\frac{2}{\epsilon}\right)^{1-d}+\left(\frac{\epsilon}{2}\right)^{d}\right) \tilde{C}_{\sigma}^{|\alpha|+|\beta|+1}|\alpha+\beta| !^{\mu}\langle x\rangle^{\delta-|\beta|}\langle\xi\rangle_{h}^{d-|\alpha|}
$$

with a new constant $\tilde{C}_{\sigma}$. As special cases we may conclude from (30) the following estimates:

$$
\begin{aligned}
& \delta=1-\sigma, d=0: \\
& \left|\partial_{\xi}^{\alpha} \partial_{x}^{\beta} \tilde{\Lambda}(t, x, \xi)\right| \leq M\left(\frac{2 T}{\epsilon}+1\right) \tilde{C}_{\sigma}^{|\alpha|+|\beta|+1}|\alpha+\beta| !^{\mu}\langle x\rangle^{1-\sigma-|\beta|}\langle\xi\rangle_{h}^{-|\alpha|} \\
& \delta=0, d=1-\sigma: \\
& \left|\partial_{\xi}^{\alpha} \partial_{x}^{\beta} \tilde{\Lambda}(t, x, \xi)\right| \leq M\left(T\left(\frac{2}{\epsilon}\right)^{\sigma}+\left(\frac{\epsilon}{2}\right)^{1-\sigma}\right) \tilde{C}_{\sigma}^{|\alpha|+|\beta|+1}|\alpha+\beta| !^{\mu}\langle x\rangle^{-|\beta|}\langle\xi\rangle_{h}^{1-\sigma-|\alpha|} \\
& \delta=-\sigma, d=1: \\
& \left|\partial_{\xi}^{\alpha} \partial_{x}^{\beta} \tilde{\Lambda}(t, x, \xi)\right| \leq M\left(T+\frac{\epsilon}{2}\right) \tilde{C}_{\sigma}^{|\alpha|+|\beta|+1}|\alpha+\beta| !^{\mu}\langle x\rangle^{-\sigma-|\beta|}\langle\xi\rangle_{h}^{1-|\alpha|}
\end{aligned}
$$

Notice the following observations: 
1. In (31), we can estimate $\left|\partial_{\xi}^{\alpha} \partial_{x}^{\beta} \Lambda(t, x, \xi)\right|$ by a constant which depends only on $M$ after choosing $\epsilon$ arbitrarily positive but then fixed, and taking the parameter $T$ small enough (i.e., $\left.\frac{2 T}{\epsilon}<1\right)$.

2. In (32), we can estimate $\left|\partial_{\xi}^{\alpha} \partial_{x}^{\beta} \Lambda(t, x, \xi)\right|$ by a constant which is independent of $M$ after taking $\epsilon$ small enough (i.e., $M\left(\frac{\epsilon}{2}\right)^{1-\sigma}<1$ ) and then the parameter $T$ small enough (i.e., $M T\left(\frac{2}{\epsilon}\right)^{\sigma}<1$ ).

3. In (33) we can estimate $\left|\partial_{\xi}^{\alpha} \partial_{x}^{\beta} \Lambda(t, x, \xi)\right|$ by a constant which is independent of $M$ after taking $T$ and $\epsilon$ small enough.

Notice, moreover, that in the intermediate case $\delta=1-\sigma-\frac{1}{s}$ and $d=\frac{1}{s}$ with $0 \leq \frac{1}{s} \leq 1-\sigma$ we get

$$
\begin{aligned}
\left|\partial_{\xi}^{\alpha} \partial_{x}^{\beta} \tilde{\Lambda}(t, x, \xi)\right| \leq & M\left(T\left(\frac{2}{\epsilon}\right)^{\sigma+\frac{1}{s}}+\left(\frac{\epsilon}{2}\right)^{\frac{1}{s}}\right) \\
& \times \tilde{C}_{\sigma}^{|\alpha|+|\beta|+1}|\alpha+\beta| !^{\mu}\langle x\rangle^{1-\sigma-\frac{1}{s}-|\beta|}\langle\xi\rangle_{h}^{\frac{1}{s}-|\alpha|},
\end{aligned}
$$

where the constants which gives the semi-norms of $\tilde{\Lambda}$ can be chosen arbitrarily small by taking $\epsilon$ and $T$ small enough.

Formula (32) states that we can consider $\tilde{\Lambda}=\tilde{\Lambda}(t, x, \xi)$ for all $t \in[0, T]$ as a symbol in $S_{\mu}^{1-\sigma}\left(\mathbb{R}^{n}\right)$ for every $\mu>1$. Moreover, $\tilde{\Lambda}$ satisfies (12) with $1-\sigma$ instead of $\frac{1}{s}$. So we can apply Theorem 6.14 in [8] and obtain that if $h$ is large enough, then the operator $\mathrm{e}^{\tilde{\Lambda}}$ is invertible on $L^{2}$ and $\left(\mathrm{e}^{\tilde{\Lambda}}\right)^{-1}$ has the form (14). This provides also the invertibility of $\mathrm{e}^{\Lambda}=\mathrm{e}^{\tilde{\Lambda}+\Lambda_{3}}=\mathrm{e}^{\tilde{\Lambda}} \mathrm{e}^{\Lambda_{3}}$ with inverse $\mathrm{e}^{-\Lambda_{3}}\left(\mathrm{e}^{\tilde{\Lambda}}\right)^{-1}$ since $\mathrm{e}^{\Lambda_{3}}$ is trivially invertible. Moreover, the conjugation $\mathrm{e}^{\Lambda}(t, x, D) p(t, x, D)\left(\mathrm{e}^{\Lambda}(t, x, D)\right)^{-1}$ makes sense and by (17) the following formula holds for every $p \in S_{1 /(1-\sigma)}^{m}\left(\mathbb{R}^{n}\right)$ :

$$
\begin{aligned}
& \mathrm{e}^{\Lambda}(t, x, D) p(t, x, D)\left(\mathrm{e}^{\Lambda}(t, x, D)\right)^{-1} \\
& \quad=\mathrm{e}^{\tilde{\Lambda}}(t, x, D)\left(\mathrm{e}^{\Lambda_{3}(t, D)} p(t, x, D) \mathrm{e}^{-\Lambda_{3}(t, D)}\right)\left(\mathrm{e}^{\tilde{\Lambda}}\right)^{-1}(t, x, D) \\
& \quad=\mathrm{e}^{\tilde{\Lambda}}(t, x, D) \operatorname{op}\left(p(t, x, \xi)+p_{1}(t, x, \xi)+p_{2}(t, x, \xi)\right)\left(\mathrm{e}^{\tilde{\Lambda}}\right)^{-1}(t, x, D) \\
& \quad=p(t, x, D)+q(t, x, D)+r(t, x, D),
\end{aligned}
$$

where

$$
\begin{aligned}
p_{1}(t, x, \xi)= & \sum_{j=1}^{n} \frac{M(T-t)}{s_{1}}\langle\xi\rangle_{h}^{\frac{1}{s_{1}}-1} \partial_{\xi_{j}}\langle\xi\rangle_{h} D_{x_{j}} p(t, x, \xi) \in S_{\frac{1}{1-\sigma}}^{m-1+\frac{1}{s_{1}}} \subset S_{\frac{1}{1-\sigma}}^{m-\sigma}, \\
p_{2}(t, x, \xi) \in & S_{\frac{1}{1-\sigma}}^{m-2\left(1-\frac{1}{s_{1}}\right)} \subset S_{\frac{1}{1-\sigma}}^{m-2 \sigma}, \\
q(t, x, \xi)= & \sum_{|\alpha|=1} \partial_{\xi}^{\alpha} p(t, x, \xi)\left(i \partial_{x}\right)^{\alpha} \tilde{\Lambda}(t, x, \xi) \\
& +\sum_{|\beta|=1} D_{x}^{\beta} p(t, x, \xi) \partial_{\xi}^{\beta} \tilde{\Lambda}(t, x, \xi)+p_{1}(t, x, \xi), \\
r(t, x, \xi) \in & S_{\frac{1}{1-\sigma}}^{m-2\left(1-\frac{1}{s_{1}}\right)} \subset S_{\frac{1}{1-\sigma}}^{m-2 \sigma} .
\end{aligned}
$$


Let us complete this section by checking that the function $\Lambda=\Lambda(t, x, \xi)$ satisfies (19). By (22), (23), (24), (25), we compute

$$
\begin{aligned}
\partial_{t} \Lambda(t, x, \xi)+ & 2 \sum_{j=1}^{n} \xi_{j} \partial_{x_{j}} \Lambda(t, x, \xi)=\partial_{t} \tilde{\Lambda}(t, x, \xi)+\partial_{t} \Lambda_{3}(t, \xi)+2 \sum_{j=1}^{n} \xi_{j} \partial_{x_{j}} \tilde{\Lambda}(t, x, \xi) \\
\leq & \partial_{t} \tilde{\Lambda}(t, x, \xi)+2 \sum_{j=1}^{n} \xi_{j} \partial_{x_{j}} \tilde{\Lambda}(t, x, \xi)=-M\langle x\rangle^{-\sigma}\langle\xi\rangle_{h}\left(1-\chi\left(\frac{\langle x\rangle}{\epsilon\langle\xi\rangle_{h}}\right)\right) \\
& +2 \sum_{j=1}^{n} \xi_{j}\left(\chi^{\prime}\left(\frac{2\langle x\rangle}{\epsilon\langle\xi\rangle_{h}}\right) 2 \epsilon^{-1}\langle\xi\rangle_{h}^{-1} \lambda(x, \xi) \partial_{x_{j}}\langle x\rangle+\chi\left(\frac{2\langle x\rangle}{\epsilon\langle\xi\rangle_{h}}\right) \partial_{x_{j}} \lambda(x, \xi)\right) \\
& +2 \sum_{j=1}^{n} M(T-t)\langle\xi\rangle_{h} \xi_{j}\left(\left(\partial_{x_{j}}\langle x\rangle^{-\sigma}\right)\left(1-\chi\left(\frac{\langle x\rangle}{\epsilon\langle\xi\rangle_{h}}\right)\right)\right. \\
& \left.+\langle x\rangle^{-\sigma} \chi^{\prime}\left(\frac{\langle x\rangle}{\epsilon\langle\xi\rangle_{h}}\right) \epsilon^{-1}\langle\xi\rangle_{h}^{-1} \partial_{x_{j}}\langle x\rangle\right)
\end{aligned}
$$

since $\partial_{t} \Lambda_{3}(t, \xi)=-M\langle\xi\rangle_{h}^{\frac{1}{s_{1}}} \leq 0$. Now we use (26), (28) and we take account of the support of $\chi, \chi^{\prime}$, to verify that all the terms of the right-hand side of the last formula (except the ones containing the partial derivative $\partial_{x_{j}} \lambda$, but to those terms we apply the estimate (26)) behave like $\langle x\rangle^{-\sigma}\langle\xi\rangle_{h}$. All these terms, except the first one, are moreover bounded by arbitrarily small constants, since we can choose $\epsilon$ small, and then $T$ small as described above. Summarizing, these considerations imply the crucial inequality (19).

\section{Proof of the main result}

Before giving the proof of Theorem 1, we will state and prove the following lemma which deals with the continuity of $\mathrm{e}^{\Lambda}$ and ${ }^{R} \mathrm{e}^{-\Lambda}$ with respect to the spaces (4) and (5) of our interest. This lemma provides the way to shift from the solution to the original Cauchy problem (20) to the solution to the equivalent (and $L^{2}$ well-posed) Cauchy problem (21) and to shift back.

Lemma 1 Let us choose $m \geq 0, \sigma \in(0,1), s_{1}, s_{2} \in\left(\frac{1}{1-\sigma}, \infty\right], s_{2} \geq s_{1}, A>0$ and $\rho>0$. Consider the function $\Lambda$ which is defined in (22). Then, for every parameters $A$ and $\rho$ satisfying the conditions

$$
A>\sup _{t \in[0, T], x, \xi \in \mathbb{R}^{n}} \frac{\tilde{\Lambda}(t, x, \xi)}{\langle x\rangle^{1-\sigma-\frac{1}{s_{2}}}\langle\xi\rangle_{h}^{\frac{1}{s_{2}}}} \text {, and } \rho>\sup _{t \in[0, T], \xi \in \mathbb{R}^{n}} \frac{\Lambda_{3}(t, \xi)}{\langle\xi\rangle_{h}^{\frac{1}{s_{1}}}}
$$

we have the following mapping properties:

1. the mapping $\mathrm{e}^{\Lambda}: \mathcal{A}_{A, \rho}^{s_{1}, s_{2}}\left(H^{m}\right) \longrightarrow H^{m}$ is continuous;

2. the mapping ${ }^{R} \mathrm{e}^{-\tilde{\Lambda}}: H^{m} \longrightarrow \mathcal{B}_{A, 0}^{s_{1}, s_{2}}\left(H^{m}\right)$ is continuous.

Proof Let us recall that the function $\tilde{\Lambda}$ satisfies (34) for every $\frac{1}{s} \leq \frac{1}{s_{1}}$ being $\frac{1}{s_{1}}<1-\sigma$. Since formula (34) holds for every $0 \leq \frac{1}{s} \leq 1-\sigma$, we take $s=s_{2}>\frac{1}{1-\sigma}$. Then we get

$$
\sup _{t \in[0, T], x, \xi \in \mathbb{R}^{n}} \frac{\tilde{\Lambda}(t, x, \xi)}{\langle x\rangle^{1-\sigma-\frac{1}{s_{2}}}\langle\xi\rangle_{h}^{\frac{1}{s_{2}}}}=M\left(T\left(\frac{2}{\epsilon}\right)^{\sigma+\frac{1}{s_{2}}}+\left(\frac{\epsilon}{2}\right)^{\frac{1}{s_{2}}}\right) \tilde{C}_{\sigma}<\infty,
$$


so the choice of the parameter $A$ is possible. On the other hand, we have

$$
\sup _{t \in[0, T], \xi \in \mathbb{R}^{n}} \frac{\Lambda_{3}(t, \xi)}{\langle\xi\rangle_{h}^{\frac{1}{s_{1}}}}=\sup _{t \in[0, T], \xi \in \mathbb{R}^{n}} M(T-t)=M T<\infty,
$$

and also the choice of the parameter $\rho$ is possible. Consider now, for $u \in \mathcal{A}_{A, \rho}^{s_{1}, s_{2}}\left(H^{m}\right)$ with $A, \rho$ as large as we need,

$$
\mathrm{e}^{\Lambda} u=\mathrm{e}^{\Lambda}\left(\mathrm{e}^{A\langle x\rangle^{1-\sigma-\frac{1}{s_{2}}}\langle D\rangle_{h}^{\frac{1}{s_{2}}}+\rho\langle D\rangle_{h}^{\frac{1}{s_{1}}}}\right)^{-1} w
$$

with

$$
w=\mathrm{e}^{A\langle x\rangle^{1-\sigma-\frac{1}{s_{2}}}\langle D\rangle_{h}^{\frac{1}{s_{2}}}+\rho\langle D\rangle_{h}^{\frac{1}{s_{1}}}} u \in H^{m} .
$$

The operator

$$
\left(\mathrm{e}^{A\langle x\rangle^{1-\sigma-\frac{1}{s_{2}}}\langle D\rangle_{h}^{\frac{1}{s_{2}}}+\rho\langle D\rangle_{h}^{\frac{1}{s_{1}}}}\right)^{-1}
$$

has the structure given by (14). Hence,

$$
\begin{aligned}
\mathrm{e}^{\Lambda} u & =\mathrm{e}^{\Lambda}\left({ }^{R} \mathrm{e}^{-A\langle x\rangle^{1-\sigma-\frac{1}{s_{2}}}\langle D\rangle_{h}^{\frac{1}{s_{2}}}-\rho\langle D\rangle_{h}^{\frac{1}{s_{1}}}}\right)(1+p) w \\
& =\mathrm{e}^{\Lambda}\left({ }^{R} \mathrm{e}^{-A\langle x\rangle^{1-\sigma-\frac{1}{s_{2}}}\langle D\rangle_{h}^{\frac{1}{s_{2}}}-\rho\langle D\rangle_{h}^{\frac{1}{s_{1}}}}\right) z,
\end{aligned}
$$

where the principal part of $p$ is

$$
\sum_{j=1}^{n} \partial_{\xi_{j}} D_{x_{j}}\left(A\langle x\rangle^{1-\sigma-\frac{1}{s_{2}}}\langle\xi\rangle_{h}^{\frac{1}{s_{2}}}+\rho\langle\xi\rangle_{h}^{\frac{1}{s_{1}}}\right) \in S^{0} .
$$

Consequently, $z=(1+p) w \in H^{m}$. Now, let us notice that

$$
\left({ }^{R} \mathrm{e}^{-A\langle\cdot\rangle^{1-\sigma-\frac{1}{s_{2}}}\langle D\rangle_{h}^{\frac{1}{s_{2}}}-\rho\langle D\rangle_{h}^{\frac{1}{s_{1}}}} u\right)(x)=\left(\left(e^{-A\langle\cdot)^{1-\sigma-\frac{1}{s_{2}}}\langle D\rangle_{h}^{\frac{1}{s_{2}}}-\rho\langle D\rangle_{h}^{\frac{1}{s_{1}}}}\right)^{*} u\right)(-x) .
$$

Indeed, using the $L^{2}$ scalar product we may compute as follows:

$$
\begin{aligned}
& \left\langle\left(\mathrm{e}^{-A\langle\cdot\rangle^{1-\sigma-\frac{1}{s_{2}}}\langle D\rangle_{h}^{\frac{1}{s_{2}}}-\rho\langle D\rangle_{h}^{\frac{1}{s_{1}}}}\right)^{*} u, v\right\rangle=\left\langle u,\left(\mathrm{e}^{-A\langle\cdot\rangle^{1-\sigma-\frac{1}{s_{2}}}}\langle D\rangle_{h}^{\frac{1}{s_{2}}}-\rho\langle D\rangle_{h}^{\frac{1}{s_{1}}}\right) v\right\rangle \\
& =\int_{\mathbb{R}^{n}} u(y)\left(\mathrm{e}^{-A\langle\cdot\rangle^{1-\sigma-\frac{1}{s_{2}}}\langle D\rangle_{h}^{\frac{1}{s_{2}}}-\rho\langle D\rangle_{h}^{\frac{1}{s_{1}}}}\right) v(y) \mathrm{d} y \\
& =\int_{\mathbb{R}^{n}} \int_{\mathbb{R}^{n}} \mathrm{e}^{-i y \xi-A\langle y\rangle^{1-\sigma-\frac{1}{s_{2}}}\langle\xi\rangle_{h}^{\frac{1}{s_{2}}}-\rho\langle\xi\rangle_{h}^{\frac{1}{s_{1}}} \overline{\hat{v}}(\xi) u(y)(2 \pi)^{-n} \mathrm{~d} \xi \mathrm{d} y} \\
& =\int_{\mathbb{R}^{n}} \int_{\mathbb{R}^{n}} \int_{\mathbb{R}^{n}} \mathrm{e}^{i x \xi-i y \xi-A\langle y\rangle^{1-\sigma-\frac{1}{s_{2}}}\langle\xi\rangle_{h}^{\frac{1}{s_{2}}}-\rho\langle\xi\rangle_{h}^{\frac{1}{s_{1}}} \bar{v}(x) u(y)(2 \pi)^{-n} \mathrm{~d} x \mathrm{~d} \xi \mathrm{d} y} \\
& =\left\langle\int_{\mathbb{R}^{n}} \int_{\mathbb{R}^{n}} \mathrm{e}^{i \cdot \xi-i y \xi-A\langle y\rangle^{1-\sigma-\frac{1}{s_{2}}}\langle\xi\rangle_{h}^{\frac{1}{s_{2}}}-\rho\langle\xi\rangle_{h}^{\frac{1}{s_{1}}}} u(y)(2 \pi)^{-n} \mathrm{~d} \xi \mathrm{d} y, v\right\rangle .
\end{aligned}
$$


This implies

$$
\begin{aligned}
\left(\mathrm{e}^{-A\langle\cdot\rangle^{1-\sigma-\frac{1}{s_{2}}}\langle D\rangle_{h}^{\frac{1}{s_{2}}}-\rho\langle D\rangle_{h}^{\frac{1}{s_{1}}}}\right)^{*} u(x) \\
=\int_{\mathbb{R}^{n}} \int_{\mathbb{R}^{n}} \mathrm{e}^{i(x-y) \xi-A\langle y\rangle^{1-\sigma-\frac{1}{s_{2}}}\langle\xi\rangle_{h}^{\frac{1}{s_{2}}}-\rho\langle\xi\rangle_{h}^{\frac{1}{s_{1}}} u(y)(2 \pi)^{-n} \mathrm{~d} \xi \mathrm{d} y} \\
=\int_{\mathbb{R}^{n}} \int_{\mathbb{R}^{n}} \mathrm{e}^{i(-x+y) \xi-A\langle y\rangle^{1-\sigma-\frac{1}{s_{2}}}\langle\xi\rangle_{h}^{\frac{1}{s_{2}}}-\rho\langle\xi\rangle_{h}^{\frac{1}{s_{1}}}} u(y)(2 \pi)^{-n} \mathrm{~d} \xi \mathrm{d} y \\
=\left({ }^{R} \mathrm{e}^{\left.-A\langle\cdot\rangle^{1-\sigma-\frac{1}{s_{2}}}\langle D\rangle_{h}^{\frac{1}{s_{2}}}-\rho\langle D\rangle_{h}^{\frac{1}{s_{1}}} u\right)(-x) .}\right.
\end{aligned}
$$

For every $A^{\prime}<A$, the symbol

$$
a(x, \xi)=\sigma\left(\left(\mathrm{e}^{-A\langle x\rangle^{1-\sigma-\frac{1}{s_{2}}}\langle D\rangle_{h}^{\frac{1}{s_{2}}}-\rho\langle D\rangle_{h}^{\frac{1}{s_{1}}}}\right)^{*}\right)(x, \xi)
$$

satisfies

$$
\left|\partial_{\xi}^{\alpha} D_{x}^{\beta} a(x, \xi)\right| \leq C\left(A^{\prime}, \alpha, \beta\right) \mathrm{e}^{-A^{\prime}\langle x\rangle^{1-\sigma-\frac{1}{s_{2}}}\langle\xi\rangle_{h}^{\frac{1}{s_{2}}}-\rho\langle\xi\rangle_{h}^{\frac{1}{s_{1}}}} .
$$

For $s_{1}, s_{2}<+\infty$, the symbol of the reversed operator is so of class $S^{-\infty}$, and the reverse turns out to be a regularizing operator in this specific case. Coming back to the composition in (39), we gain that the composition is well defined, and the symbol $q=q(x, \xi)$ of the composed operator satisfies the estimate

$$
\left|\partial_{\xi}^{\alpha} D_{x}^{\beta} q(x, \xi)\right| \leq C\left(A^{\prime}, \alpha, \beta\right)\langle\xi\rangle^{-\sigma|\alpha|} e^{\Lambda(t, x, \xi)-A^{\prime}\langle x\rangle^{1-\sigma-\frac{1}{s_{2}}}\langle\xi\rangle_{h}^{\frac{1}{s_{2}}}-\rho\langle\xi\rangle_{h}^{\frac{1}{s_{1}}}} .
$$

Indeed, with $A^{\prime}$ and $\rho$ large enough it follows

$$
\begin{aligned}
& \tilde{\Lambda}(t, x, \xi)-A^{\prime}\langle x\rangle^{1-\sigma-\frac{1}{s_{2}}}\langle\xi\rangle_{h}^{\frac{1}{s_{2}}} \\
& \leq\left(M\left(T\left(\frac{2}{\epsilon}\right)^{\sigma+\frac{1}{s_{2}}}+\left(\frac{\epsilon}{2}\right)^{\frac{1}{s_{2}}}\right) \tilde{C}_{\sigma}-A^{\prime}\right)\langle x\rangle^{1-\sigma-\frac{1}{s_{2}}}\langle\xi\rangle_{h}^{\frac{1}{s_{2}}} \\
& =-C_{T}\langle x\rangle^{1-\sigma-\frac{1}{s_{2}}}\langle\xi\rangle_{h}^{\frac{1}{s_{2}}}, \\
& \Lambda_{3}(t, \xi)-\rho\langle\xi\rangle_{h}^{\frac{1}{s_{1}}} \leq(M T-\rho)\langle\xi\rangle_{h}^{\frac{1}{s_{1}}}=-C_{T}^{\prime}\langle\xi\rangle_{h}^{\frac{1}{s_{1}}} .
\end{aligned}
$$

Summing up,

$$
\mathrm{e}^{\Lambda}(t, x, D)\left({ }^{R} \mathrm{e}^{-A\langle x\rangle^{1-\sigma-\frac{1}{s_{2}}}\langle D\rangle_{h}^{\frac{1}{s_{2}}}-\rho\langle D\rangle_{h}^{\frac{1}{s_{1}}}}\right)
$$

is a pseudo-differential operator of order zero acting on $z \in H^{m}$. So, if $u \in \mathcal{A}_{A, \rho}^{s_{1}, s_{2}}\left(H^{m}\right)$ with $A$ and $\rho$ large enough, then $\mathrm{e}^{\Lambda} u \in H^{m}$. Similarly, one obtains that for every $u \in H^{m}$ the function ${ }^{R} \mathrm{e}^{-\tilde{\Lambda}} u \in \mathcal{B}_{A, 0}^{s_{1}, s_{2}}\left(H^{m}\right)$ has the property

$$
\mathrm{e}^{-A\langle x\rangle^{1-\sigma-\frac{1}{s_{2}}}\langle D\rangle_{h}^{\frac{1}{s_{2}}} R} \mathrm{e}^{-\tilde{\Lambda}} u \in H^{m} .
$$

This completes the proof. 
Proof of Theorem 1 Let us perform the change of variables

$$
v(t, x)=\mathrm{e}^{\Lambda(t, x, D)} u(t, x),
$$

with $\Lambda$ as in (22). Take the parameter $h$ large so that $\mathrm{e}^{\Lambda}$ is invertible and the conjugation formula (35) holds. By this change of variables, the Cauchy problem (20) is reduced to the equivalent Cauchy problem (21). In the further considerations we are going to show that the remainder $r_{0}$ is of order zero and the operator $A_{\Lambda}$ satisfies

$$
2 \Re\left\langle A_{\Lambda}(t, x, D) v, v\right\rangle \geq 0 .
$$

By (22) and (35), we have

$$
\begin{aligned}
S_{\Lambda}= & \mathrm{e}^{\Lambda} S\left(\mathrm{e}^{\Lambda}\right)^{-1} \\
= & D_{t}-\Delta_{x}+i \partial_{t} \Lambda(t, x, D)+\mathrm{e}^{\Lambda} \sum_{j=1}^{n}\left(\left(\partial_{x_{j}} \Lambda\right)^{2}+\partial_{x_{j}}^{2} \Lambda+2\left(\partial_{x_{j}} \Lambda\right) \partial_{x_{j}}\right)\left(\mathrm{e}^{\Lambda}\right)^{-1} \\
& +\mathrm{e}^{\Lambda}\left(\sum_{j=1}^{n} a_{j}(t, x) D_{x_{j}}+b(t, x)\right)\left(\mathrm{e}^{\Lambda}\right)^{-1} .
\end{aligned}
$$

By formula (30) with $\delta=1-\sigma$ and $d=0$, and since $\Lambda_{3}$ does not depend on $x$, we get $\partial_{x_{j}} \Lambda, \partial_{x_{j}}^{2} \Lambda \in S_{\mu}^{0}$ for an arbitrary $\mu>1$. After applying formula (35) and taking account of the Gevrey regularity of the coefficients $a_{j}, b$ we arrive at

$$
\begin{aligned}
S_{\Lambda}= & D_{t}-\Delta_{x}+i \partial_{t} \Lambda(t, x, D) \\
& +\mathrm{e}^{\Lambda}\left(\sum_{j=1}^{n} 2 i\left(\partial_{x_{j}} \Lambda\right) D_{x_{j}}+\sum_{j=1}^{n} a_{j}(t, x) D_{x_{j}}+b(t, x)\right)\left(\mathrm{e}^{\Lambda}\right)^{-1}+r(t, x, D) \\
= & D_{t}-\Delta_{x}+i \partial_{t} \Lambda(t, x, D)+\sum_{j=1}^{n} 2 i\left(\partial_{x_{j}} \Lambda\right) D_{x_{j}}+\sum_{j=1}^{n} a_{j}(t, x) D_{x_{j}} \\
& +r_{1-\sigma}(t, x, D)+r_{0}(t, x, D),
\end{aligned}
$$

where $r=r(t, x, \xi)$ and $r_{0}=r_{0}(t, x, \xi)$ are symbols in $S_{\frac{1}{1-\sigma}}^{0}$. Moreover, $r_{1}=r_{1-\sigma}(t, x, \xi)$ is a symbol of positive order with principal part given by

$$
\sum_{j=1}^{n} \sum_{|\beta|=1}\left(D_{x}^{\beta} a_{j}(t, x)\right) \partial_{\xi}^{\beta} \Lambda(x, \xi) \xi_{j} \in S_{\frac{1}{1-\sigma}}^{1-\sigma}
$$

by using our assumption $\frac{1}{s_{1}}<1-\sigma$. Here, we also use that the symbols

$$
\begin{aligned}
& \sum_{|\alpha|=1} \partial_{\xi}^{\alpha}\left(\left(\partial_{x_{j}} \Lambda\right) \xi_{j}\right)\left(i \partial_{x}\right)^{\alpha} \Lambda(x, \xi)+\sum_{|\beta|=1} D_{x}^{\beta}\left(\left(\partial_{x_{j}} \Lambda\right) \xi_{j}\right) \partial_{\xi}^{\beta} \Lambda(x, \xi), \\
& \sum_{|\alpha|=1} \partial_{\xi}^{\alpha}\left(a_{j} \xi_{j}\right)\left(i \partial_{x}\right)^{\alpha} \Lambda(x, \xi)
\end{aligned}
$$

belong to $S_{\frac{1}{1-\sigma}}^{0}$ as well by choosing $\delta=1-\sigma$ and $d=0$ in (30) and by taking into consideration the structure of $\Lambda_{3}$. Consequently, we get

$$
S_{\Lambda}=D_{t}-\Delta_{x}-i A_{\Lambda}(t, x, D)+r_{0}(t, x, D)
$$


with

$$
A_{\Lambda}(t, x, \xi)=-\partial_{t} \Lambda(t, x, \xi)-\sum_{j=1}^{n} 2\left(\partial_{x_{j}} \Lambda\right) \xi_{j}+i \sum_{j=1}^{n} a_{j}(t, x) \xi_{j}+i r_{1-\sigma}(t, x, \xi) .
$$

Now we look for an energy estimate for $v=v(t, x)$. We compute

$$
\begin{aligned}
\frac{\mathrm{d}}{\mathrm{d} t} & \|v(t, \cdot)\|_{L^{2}}^{2}=2 \Re\left\langle v^{\prime}, v\right\rangle_{L^{2}} \\
& =2 \Re\langle i \Delta v, v\rangle_{L^{2}}-2 \Re\left\langle A_{\Lambda}(t, x, D) v, v\right\rangle_{L^{2}}-2 \Re\left\langle\left(i r_{0}\right) v, v\right\rangle_{L^{2}} \\
& \leq C\|v(t, \cdot)\|_{L^{2}}^{2}-2 \Re\left\langle A_{\Lambda}(t, x, D) v, v\right\rangle_{L^{2}} \\
& \leq C\|v(t, \cdot)\|_{L^{2}}^{2}-\left\langle\left(A_{\Lambda}+A_{\Lambda}^{*}\right)(t, x, D) v, v\right\rangle_{L^{2}} .
\end{aligned}
$$

Taking account of (19), (3), (33) and (29) with $\delta=1-\sigma$ and $d=0$, we obtain

$$
\begin{aligned}
\left(A_{\Lambda}+\left(A_{\Lambda}\right)^{*}\right)(t, x, \xi)=-2\left(\partial_{t} \Lambda(t, x, \xi)+\sum_{j=1}^{n} 2\left(\partial_{x_{j}} \Lambda\right) \xi_{j}\right)-2 \sum_{j=1}^{n} \Im a_{j}(t, x) \xi_{j} \\
\quad+\left(i r_{1-\sigma}+i r_{1-\sigma}^{*}\right)+\text { terms of order zero } \\
\geq 2 M\langle x\rangle^{-\sigma}\langle\xi\rangle_{h}-2 C\langle x\rangle^{-\sigma}\langle\xi\rangle_{h}-2 C_{\sigma} M T\langle x\rangle^{-\sigma}\langle\xi\rangle_{h}-2 C_{\sigma} M\langle x\rangle^{1-\sigma} \chi\left(\frac{2\langle x\rangle}{\epsilon\langle\xi\rangle_{h}}\right) \\
\geq 2 M\langle x\rangle^{-\sigma}\langle\xi\rangle_{h}-2 C\langle x\rangle^{-\sigma}\langle\xi\rangle_{h}-2 C_{\sigma} M T\langle x\rangle^{-\sigma}\langle\xi\rangle_{h}-2 C_{\sigma} M \epsilon\langle x\rangle^{-\sigma}\langle\xi\rangle_{h} \\
\geq 2\left(M-C-C_{\sigma} M T-C_{\sigma} M \epsilon\right)\langle x\rangle^{-\sigma}\langle\xi\rangle_{h},
\end{aligned}
$$

where we have also used that $\langle x\rangle \leq \epsilon\langle\xi\rangle_{h}$ on the support of $\Lambda_{2}$. First we choose $M>C+2$, where $C$ is the constant in (3). Then, we choose $\epsilon$ and $T$ so small that $C_{\sigma} M \epsilon<1$ and $C_{\sigma} M T<1$. With these choices, we have

$$
\left(A_{\Lambda}+\left(A_{\Lambda}\right)^{*}\right)(t, x, \xi) \geq 2(M-C-2)\langle x\rangle^{-\sigma}\langle\xi\rangle_{h} \geq 0 .
$$

Applying the sharp Gårding inequality we obtain $2 \Re\left\langle A_{\Lambda}(t, x, D) v, v\right\rangle \geq 0$. Hence,

$$
\frac{\mathrm{d}}{\mathrm{d} t}\|v(t, \cdot)\|_{L^{2}}^{2} \leq C\|v(t, \cdot)\|_{L^{2}}^{2}
$$

Thus, the energy estimate

$$
\|v(t, \cdot)\|_{L^{2}}^{2} \leq c\left\|g_{\Lambda}\right\|_{L^{2}}^{2},
$$

is established for all $t \in[0, T]$ with a suitable positive constant $c$. The Cauchy problem for $v$ is so well-posed in $L^{2}$.

It is well-posed also in Sobolev spaces $H^{m}$, since the conjugation $\langle D\rangle^{m} S_{\Lambda}\langle D\rangle^{-m}$ transforms the Cauchy problem $S_{\Lambda} v=0, v(0, x)=g_{\Lambda}(x)$ with $g_{\Lambda} \in H^{m}$ to an equivalent Cauchy problem $\tilde{S}_{\Lambda} \tilde{v}=0, \tilde{v}(0, x)=\tilde{g}_{\Lambda}(x)$ with $\tilde{g}_{\Lambda} \in L^{2}$, where $\tilde{v}=\langle D\rangle^{m} v$ and a new pseudo-differential operator $\tilde{S}_{\Lambda}$ which has exactly the same structure as $S_{\Lambda}$.

To go back to the solution $u$ to the original Cauchy problem, notice that $g \in \mathcal{A}_{A, \rho}^{s_{1}, s_{2}}\left(H^{m}\right)$ implies by Lemma 1 that we can obtain $g_{\Lambda}=\mathrm{e}^{\Lambda(0)} g \in H^{m}$ by a sharp choice of $M, \epsilon, \rho$ and $T^{*} \leq T$. The Cauchy problem (21) is $H^{m}$ well-posed, so it admits a unique solution $v \in C\left(\left[0, T^{*}\right], H^{m}\right)$. For every $t \in\left[0, T^{*}\right], v(t, \cdot) \in H^{m}$ implies by Lemma 1 that 


$$
\begin{aligned}
u(t, \cdot) & =\left(\left(\mathrm{e}^{\tilde{\Lambda}} \mathrm{e}^{\Lambda_{3}}\right)^{-1} v\right)(t, \cdot)=\left(\mathrm{e}^{-\Lambda_{3}}\left(\mathrm{e}^{\tilde{\Lambda}}\right)^{-1} v\right)(t, \cdot) \\
& =\mathrm{e}^{-M(T-t)\left\langle D_{x}\right\rangle^{\frac{1}{s_{1}}}}\left(\left(\mathrm{e}^{\tilde{\Lambda}}\right)^{-1} v\right)(t, \cdot) \\
& =\mathrm{e}^{-M(T-t)\left\langle D_{x}\right\rangle^{\frac{1}{s_{1}}}}\left(\left(R^{-\tilde{\Lambda}}\right)(1+p) v\right)(t, \cdot) \\
& =\mathrm{e}^{-M(T-t)\left\langle D_{x}\right\rangle^{\frac{1}{s_{1}}}} R \mathrm{e}^{-\tilde{\Lambda}} z(t, \cdot) \\
& =\mathrm{e}^{-M(T-t)\left\langle D_{x}\right\rangle^{\frac{1}{s_{1}}}} w(t, \cdot) \in \mathcal{B}_{A, \rho^{\prime}}^{s_{1}, s}\left(H^{m}\right)
\end{aligned}
$$

for every $0 \leq \frac{1}{s} \leq \frac{1}{s_{1}}$ and with $\rho^{\prime}=M(T-t)$, since the principal part of $p$ is in $S^{0}$, and so $z=(1+p) v \in H^{m}$. This implies $w \in \mathcal{B}_{A, 0}^{s_{1}, s}$. The proof is complete, since starting from data in $\mathcal{A}_{A, \rho}^{s_{1}, s_{2}}\left(H^{m}\right)$ we have obtained a solution $u \in \mathcal{B}_{A, \rho^{\prime}}^{s_{1}, s}\left(H^{m}\right)$ for every $0 \leq \frac{1}{s} \leq \frac{1}{s_{1}}$ and for a suitable $\rho^{\prime}$. Moreover, we may conclude for every $s \geq s_{1}$ as follows:

$$
\begin{aligned}
&\|u(t, \cdot)\|_{\mathcal{B}_{A, \rho^{\prime}}^{s_{1}, s}\left(H^{m}\right)}=\left\|\mathrm{e}^{-A\langle x\rangle^{1-\sigma-\frac{1}{s}}\langle D\rangle^{\frac{1}{s}}+\rho^{\prime}\langle D\rangle^{\frac{1}{s_{1}}}} u(t, \cdot)\right\|_{H^{m}} \\
& \quad=\left\|\mathrm{e}^{-A\langle x\rangle^{1-\sigma-\frac{1}{s}}\langle D\rangle^{\frac{1}{s}}+\rho^{\prime}\langle D\rangle^{\frac{1}{s_{1}}}} \mathrm{e}^{-\Lambda_{3}(t, D)}\left(\mathrm{e}^{\tilde{\Lambda}}\right)^{-1} v(t, \cdot)\right\|_{H^{m}} \\
& \quad=\left\|\mathrm{e}^{-A\langle x\rangle^{1-\sigma-\frac{1}{s}}\langle D\rangle^{\frac{1}{s}}}\left(\mathrm{e}^{\tilde{\Lambda}}\right)^{-1} v(t, \cdot)\right\|_{H^{m}} \\
& \quad=\left\|\left(\mathrm{e}^{\tilde{\Lambda}}\right)^{-1} v(t, \cdot)\right\|_{\mathcal{B}_{A, 0}^{s_{1}, s}\left(H^{m}\right)} \\
& \quad \leq C_{t}\|v(t, \cdot)\|_{H^{m}} \quad(\text { by continuity, see Lemma 1) } \\
& \leq C_{t}^{\prime}\left\|g_{\Lambda}\right\|_{H^{m}} \quad(\text { by the energy estimate }) \\
&=C_{t}^{\prime}\left\|\mathrm{e}^{\Lambda(0)} g\right\|_{H^{m}} \\
&=C_{t}^{\prime}\|g\|_{\mathcal{A}_{A, \rho^{\prime}}^{s_{1}, s_{2}}\left(H^{m}\right) \quad \text { (by continuity, see Lemma 1) }}
\end{aligned}
$$

with continuous functions $C_{t}, C_{t}^{\prime}$ with respect to time thanks to the well-posedness of the auxiliary Cauchy problem.

Remark 7 We remark that the choice of the parameters $A, \rho$ and $T$, depending on formulas (37) and (38), may be interpreted in two different ways:

- on the one hand, if one aims to obtain a solution defined on the whole interval $[0, T]$, then one has to choose large $A$ and $\rho$, i.e., one asks for more regularity to the data $g$;

- on the other hand, if one has a fixed regularity for the data $g$, i.e., if $A$ and $\rho$ are fixed, then one can obtain a solution in $H^{m}$ only for small times $t \in\left[0, T^{*}\right], T^{*} \leq T$.

Proof of Corollary 1 The change of variables

$$
v(t, x)=\mathrm{e}^{\Lambda(t, x, D)} u(t, x),
$$

with $\Lambda=\tilde{\Lambda}$ in (22) (i.e., $\Lambda_{3} \equiv 0$ ) and $h$ large enough to get invertibility of $\mathrm{e}^{\Lambda}$ reduces the Cauchy problem (20) to an equivalent Cauchy problem (21) with $2 \Re\left\langle A_{\Lambda}(t, x, D) v, v\right\rangle \geq 0$ and $r_{0}$ of order zero, following the same computations as in the proof of Theorem 1 .

To go back to the solution $u$ to the original Cauchy problem, notice that the assumption $\mathrm{e}^{A\langle x\rangle^{1-\sigma}} g \in H^{m}$ implies by (32) that $g_{\Lambda}=\mathrm{e}^{\Lambda(0)} g$ satisfies $\mathrm{e}^{(A-2 M)\langle x\rangle^{1-\sigma}} g_{\Lambda} \in H^{m}$. By the change of variables $w=\mathrm{e}^{(A-2 M)\langle x\rangle^{1-\sigma}} v$ we get the equivalent Cauchy problem

$$
S_{\Lambda}^{\prime} w:=\mathrm{e}^{(A-2 M)\langle x\rangle^{1-\sigma}} S_{\Lambda} \mathrm{e}^{(-A+2 M)\langle x\rangle^{1-\sigma}} w=0, \quad w(0, x)=g_{\Lambda}^{\prime}(x)
$$


with $g_{\Lambda}^{\prime} \in H^{m}$ with $S_{\Lambda}^{\prime}$ having the same structure as $S_{\Lambda}$. Consequently, the Cauchy problem for $w$ admits a unique solution $w \in C\left([0, T] ; H^{m}\right)$. The Cauchy problem for $v$ admits a unique solution satisfying $\mathrm{e}^{(A-2 M)\langle x\rangle^{1-\sigma}} v(t, \cdot) \in H^{m}$, respectively. Finally, the unique solution $u=\left(\mathrm{e}^{\Lambda}\right)^{-1} v$ of the original Cauchy problem satisfies $\mathrm{e}^{(A-4 M)\langle x\rangle^{1-\sigma}} u(t, \cdot) \in H^{m}$ for every $t \in[0, T]$. For this reason, $u(t, \cdot)$ may belong to a weighted Sobolev space with exponentially decreasing weight, compare with [2,6]. Finally, the solution $u$ satisfies the following energy estimate:

$$
\begin{aligned}
& \left\|\mathrm{e}^{(A-4 M)\langle x\rangle^{1-\sigma}} u(t, \cdot)\right\|_{H^{m}}=\left\|\mathrm{e}^{(A-4 M)\langle x\rangle^{1-\sigma}}\left(\mathrm{e}^{\Lambda}\right)^{-1} v(t, \cdot)\right\|_{H^{m}} \\
& \quad \leq C_{t}\left\|\mathrm{e}^{(A-2 M)\langle x\rangle^{1-\sigma}} v(t, \cdot)\right\|_{H^{m}}=\|w(t, \cdot)\|_{H^{m}} \\
& \quad \leq C_{t}\left\|g_{\Lambda}^{\prime}\right\|_{H^{m}}=C_{t}\left\|\mathrm{e}^{(A-2 M)\langle x\rangle^{1-\sigma}} g_{\Lambda}\right\|_{H^{m}} \\
& \quad=C_{t}\left\|\mathrm{e}^{(A-2 M)\langle x\rangle^{1-\sigma}} \mathrm{e}^{\Lambda(0)} g\right\|_{H^{m}} \\
& \quad \leq C_{t}\left\|\mathrm{e}^{(A-4 M)\langle x\rangle^{1-\sigma}} g\right\|_{H^{m}},
\end{aligned}
$$

where the function $C_{t}$ is continuous on $[0, T]$ and may change from line to line. This completes the proof.

Remark 8 The choice $\delta=1-\sigma$ and $d=0$ allows us to obtain in Corollary 1 a solution which is valued in Sobolev spaces. Notice that if $1-\sigma=\frac{1}{s}$, then to ensure $\mathrm{e}^{\Lambda(0)} g \in H^{m}$ under the assumption $\mathrm{e}^{A\langle x\rangle^{1-\sigma}} g \in H^{m}$ we need to require $C_{T} \leq A$, that is, $T$ is small enough. This is the reason why we obtain local (in time) results for the Cauchy problem for $S$. We remark that in this paper in the definition of $\Lambda_{1}$ we take the time-dependent function $\rho=\rho(t)=M(T-t)$ since we are looking for a local (in time) well-posedness result in the critical case $1-\sigma=\frac{1}{s}$, too. In the non-critical case $1-\sigma<\frac{1}{s}$, the condition $C_{T} \leq A$ is no more required. By taking the same function $\rho=\rho(t)$ as in [7], we can obtain global (in time) well-posedness of the Cauchy problem for $S$ under the assumptions of Corollary 1.

Remark 9 Let us characterize the spaces $\mathcal{A}_{A, \rho}^{s_{1}, s_{2}}\left(H^{m}\right)$ which are used in the formulation of the main results in Theorem 1, Corollaries 1 and 2. Here, $A$ and $\rho$ are positive constants, the parameter $m \geq 0$. We turn to $\mathcal{A}_{A, \rho}^{s_{1}, s_{2}}\left(H^{m}\right)$, where $\sigma \in(0,1)$ and $s_{1}, s_{2} \in\left(\frac{1}{1-\sigma}, \infty\right]$. Then due to (4)

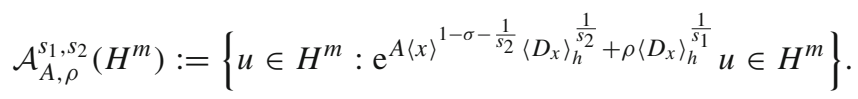

Let us introduce

$$
v:=\mathrm{e}^{\rho\left\langle D_{x}\right\rangle_{h}^{\frac{1}{s_{1}}}} u \text { with a given } u \in H^{m}, m \geq 0 .
$$

Then $v$ belongs to the Gevrey-Sobolev space

$$
H^{m, s_{1}}=\bigcup_{\rho>0} H_{\rho}^{m, s_{1}}, \text { where } H_{\rho}^{m, s_{1}}=\mathrm{e}^{-\rho\langle D\rangle^{\frac{1}{s_{1}}}} H^{m} .
$$

We apply to elements of this space the pseudo-differential operator of infinite order

$$
\mathrm{e}^{A\langle x\rangle^{1-\sigma-\frac{1}{s_{2}}}\left\langle D_{x}\right\rangle_{h}^{\frac{1}{s_{2}}}} \text { with } \frac{1}{s_{2}} \in[0,1-\sigma] .
$$

If $s_{2}=\infty$, then we apply

$$
\mathrm{e}^{A\langle x\rangle^{1-\sigma}}
$$


only, that is, $u$ belongs to the Gelfand-Shilov space

$$
S_{\frac{1}{1-\sigma}}^{S_{1}}=\left\{f \in C^{\infty}\left(\mathbb{R}^{n}\right): \sup _{x \in \mathbb{R}^{n}, \alpha \in \mathbb{N}^{n}} C^{-|\alpha|} \alpha !^{-S_{1}} \mathrm{e}^{\epsilon|x|^{1-\sigma}}\left|\partial_{x}^{\alpha} f(x)\right|<\infty\right\}
$$

with positive constants $C$ and $\epsilon$. These spaces can be characterized in the following way, too:

$$
\mathcal{S}_{\frac{1}{1-\sigma}}^{s_{1}}=\bigcup_{m_{j} \in \mathbb{R}, \rho_{j} \in \mathbb{R}^{+}, j=1,2}\left\{u \in \mathscr{S}^{\prime}\left(\mathbb{R}^{n}\right):\langle\cdot\rangle^{m_{2}}\langle D\rangle^{m_{1}} \mathrm{e}^{\rho_{2}\langle\cdot\rangle^{1-\sigma}} \mathrm{e}^{\rho_{1}\langle D\rangle^{1 / s_{1}}} u \in L^{2}\right\} .
$$

If $s_{2}=\frac{1}{1-\sigma}$, then we apply

$$
\mathrm{e}^{A\left\langle D_{x}\right\rangle_{h}^{1-\sigma}}
$$

only, that is, $u$ belongs to the Gevrey-Sobolev space $H^{1-\sigma, m}$. Let us understand the intermediate situation.

To describe the space $\mathcal{A}_{A^{\prime}, \rho^{\prime}}^{s_{1}, s_{2}}\left(H^{m}\right), A^{\prime}, \rho^{\prime}>0$, by Fourier multipliers, we use Remark 5 , (40) and the Fourier transform to get

$$
\begin{aligned}
\mathcal{A}_{A^{\prime}, \rho^{\prime}}^{s_{1}, s_{2}}\left(H^{m}\right) & =\left\{u \in H^{m}: \mathrm{e}^{\Lambda^{\prime}} u \in H^{m}\right\}=\left\{u=\left(\mathrm{e}^{\Lambda^{\prime}}\right)^{-1} w: w \in H^{m}\right\} \\
& =\left\{u={ }^{R} \mathrm{e}^{-\Lambda^{\prime}}(1+p) w: w \in H^{m}\right\}=\left\{u={ }^{R} \mathrm{e}^{-\Lambda^{\prime}} w: w \in H^{m}\right\} \\
& =\left\{u=\left(\mathrm{e}^{-\Lambda^{\prime}}\right)^{*} w: w \in H^{m}\right\}
\end{aligned}
$$

with

$$
\sigma\left(\Lambda^{\prime}\right)(x, \xi)=A^{\prime}\langle x\rangle^{1-\sigma-\frac{1}{s_{2}}}\langle\xi\rangle_{h}^{\frac{1}{s_{2}}}+\rho^{\prime}\langle\xi\rangle_{h}^{\frac{1}{s_{1}}}
$$

as in (15). Here, $w$ is a function in $H^{m}$ that may change from line to line. Now, we see that $\left(\mathrm{e}^{-\Lambda^{\prime}}\right)\left(x, D_{x}\right)$ is at least an operator of finite order. So by asymptotically developing the symbol of the adjoint we obtain

$$
\sigma\left(\left(\mathrm{e}^{-\Lambda^{\prime}}\right)^{*}(x, \xi)\right)=\mathrm{e}^{-\Lambda^{\prime \prime}(x, \xi)} \tilde{p}(x, \xi), \quad \Lambda^{\prime \prime}(x, \xi)=A^{\prime \prime}\langle x\rangle^{1-\sigma-\frac{1}{s_{2}}}\langle\xi\rangle_{h}^{\frac{1}{s_{2}}}+\rho^{\prime \prime}\langle\xi\rangle_{h}^{\frac{1}{s_{1}}},
$$

with $\tilde{p}\left(x, D_{x}\right)$ a bounded operator of order 0 and with suitable $A^{\prime \prime}, \rho^{\prime \prime}>0$. For this reason, we can characterize the space as follows: $\mathcal{A}_{A^{\prime}, \rho^{\prime}}^{s_{1}, s_{2}}\left(H^{m}\right)$ is contained in the space of all functions $u \in H^{m}$ such that

$$
u=\operatorname{op}\left(\mathrm{e}^{-\Lambda^{\prime \prime}(x, \xi)}\right)\left(x, D_{x}\right) w \text { with } w \in H^{m},
$$

where op $\left(\mathrm{e}^{-\Lambda^{\prime \prime}(x, \xi)}\right)\left(x, D_{x}\right)$ is the pseudo-differential operator of infinite order with symbol $\mathrm{e}^{-\Lambda^{\prime \prime}(x, \xi)}$.

Remark 10 Let us characterize the spaces $\mathcal{B}_{A, \rho}^{s_{1}, s_{2}}\left(H^{m}\right)$ which are used in the formulation of the main results in Theorem 1, Corollaries 1 and 2. Here, $A$ and $\rho$ are positive constants, the parameter $m \geq 0$. We turn to $\mathcal{B}_{A, \rho}^{s_{1}, s_{2}}\left(H^{m}\right)$, where $\sigma \in(0,1), s_{1} \in\left(\frac{1}{1-\sigma}, \infty\right]$ and $s_{2} \in\left[s_{1}, \infty\right]$. Then due to (5), we have

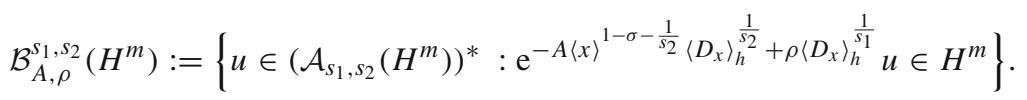

Let us introduce

$$
v:=\mathrm{e}^{\rho\left\langle D_{x}\right\rangle_{h}^{\frac{1}{s_{1}}}} u \text { with a given } u \in H^{m}, m \geq 0 .
$$


Then $v$ belongs to the Gevrey-Sobolev space $H^{s_{1}, m}$. We apply to elements of this space the pseudo-differential operator of infinite order

$$
\mathrm{e}^{-A\langle x\rangle^{1-\sigma-\frac{1}{s_{2}}}\left\langle D_{x}\right\rangle_{h}^{\frac{1}{s_{2}}}} \text { with } \frac{1}{s_{2}} \in\left[0, \frac{1}{s_{1}}\right] \text {. }
$$

If $s_{2}=\infty$, then we apply

$$
\mathrm{e}^{-A\langle x\rangle^{1-\sigma}}
$$

only, that is, $u$ belongs to a weighted Gevrey-Sobolev space with an exponentially decreasing weight. If $s_{2}=s_{1}$, then

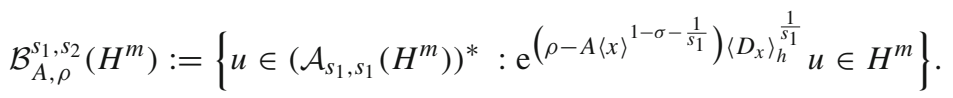

To characterize the spaces $\mathcal{B}_{A^{\prime}, \rho^{\prime}}^{s_{1}, S_{2}}\left(H^{m}\right), A^{\prime}, \rho^{\prime}>0$, by Fourier multipliers we can formally repeat the same computations done in Remark 9. By using the symbol

$$
\Lambda^{\prime}(x, \xi)=-A^{\prime}\langle x\rangle^{1-\sigma-\frac{1}{s_{2}}}\langle\xi\rangle_{h}^{\frac{1}{s_{2}}}+\rho^{\prime}\langle\xi\rangle_{h}^{\frac{1}{s_{1}}}
$$

straight-forward computations give that $\mathcal{B}_{A^{\prime}, \rho^{\prime}}^{s_{1}, s_{2}}\left(H^{m}\right)$ is contained in the space of all $u \in$ $\left(\mathcal{A}_{s_{1}, s_{2}}\left(H^{m}\right)\right)^{*}$ such that

$$
u=\operatorname{op}\left(\mathrm{e}^{-\Lambda^{\prime \prime}(x, \xi)}\right) w \text { with } w \in H^{m},
$$

where

$$
\Lambda^{\prime \prime}(x, \xi)=-A^{\prime \prime}\langle x\rangle^{1-\sigma-\frac{1}{s_{2}}}\langle\xi\rangle_{h}^{\frac{1}{s_{2}}}+\rho^{\prime \prime}\langle\xi\rangle_{h}^{\frac{1}{s_{1}}},
$$

with suitable $A^{\prime \prime}, \rho^{\prime \prime}>0$ and where op $\left(\mathrm{e}^{-\Lambda^{\prime \prime}(x, \xi)}\right)\left(x, D_{x}\right)$ is the pseudo-differential operator of infinite order with symbol $\mathrm{e}^{-\Lambda^{\prime \prime}(x, \xi)}$.

Let us now restrict to the case $s_{2}>s_{1}$. Taking into consideration

$$
\langle x\rangle^{1-\sigma-\frac{1}{s_{2}}}\langle\xi\rangle_{h}^{\frac{1}{s_{2}}} \leq C_{\varepsilon}\left(\langle x\rangle^{\left(1-\sigma-\frac{1}{s_{2}}\right) \frac{1+\varepsilon}{\varepsilon}}+\langle\xi\rangle_{h}^{\frac{1}{s_{2}}(1+\varepsilon)}\right)
$$

for all $\varepsilon>0$, a sufficiently small positive $\varepsilon$ allows to conclude from

$$
\begin{aligned}
& \mathrm{e}^{A^{\prime \prime} C_{\varepsilon}\left(\langle x\rangle^{\left(1-\sigma-\frac{1}{s_{2}}\right) \frac{1+\varepsilon}{\varepsilon}}+\langle\xi\rangle_{h}^{\frac{1}{S_{2}}(1+\varepsilon)}\right)}-\mathrm{e}^{-A^{\prime \prime} C_{\varepsilon}\left(\langle x\rangle^{\left(1-\sigma-\frac{1}{s_{2}}\right) \frac{1+\varepsilon}{\varepsilon}}+\langle\xi\rangle_{h}^{\frac{1}{s_{2}}(1+\varepsilon)}\right)} \mathrm{e}^{-\Lambda^{\prime \prime}(x, \xi)} \\
& =\mathrm{e}^{A^{\prime \prime} C_{\varepsilon}\langle x\rangle^{\left(1-\sigma-\frac{1}{s_{2}}\right) \frac{1+\varepsilon}{\varepsilon}}} \mathrm{e}^{-\rho^{\prime \prime}\langle\xi\rangle_{h}^{\frac{1}{s_{1}}}+A^{\prime \prime} C_{\varepsilon}\langle\xi\rangle_{h}^{\frac{1}{S_{2}}(1+\varepsilon)}} \\
& \quad \cdot \mathrm{e}^{-A^{\prime \prime} C_{\varepsilon}\left(\langle x\rangle^{\left(1-\sigma-\frac{1}{s_{2}}\right) \frac{1+\varepsilon}{\varepsilon}}+\langle\xi\rangle_{h}^{\frac{1}{s_{2}}(1+\varepsilon)}\right)+A^{\prime \prime}\langle x\rangle^{1-\sigma-\frac{1}{s_{2}}}\langle\xi\rangle_{h}^{\frac{1}{s_{2}}}}
\end{aligned}
$$

that $\mathcal{B}_{A^{\prime}, \rho^{\prime}}^{s_{1}, s_{2}}\left(H^{m}\right)$ is contained in the space of functions $u \in H_{\mathrm{loc}}^{m}$ such that

$$
u=\mathrm{e}^{\tilde{A}\langle x\rangle^{\left(1-\sigma-\frac{1}{s_{2}}\right) \frac{1+\varepsilon}{\varepsilon}}} \mathrm{e}^{-\tilde{\rho}\left\langle D_{x}\right\rangle_{h}^{\frac{1}{s_{1}}}} w \text { with } w \in H^{m}
$$

for suitable positive constants $\tilde{A}$ and $\tilde{\rho}$. Consequently, $u$ belongs to a Gevrey space with exponentially decaying weight. 


\title{
Compliance with ethical standards
}

Conflict of interest The authors declare that they have no conflict of interest.

\section{References}

1. Ascanelli, A., Cappiello, M.: Weighted energy estimates for p-evolution equations in SG classes. J. Evol. Equ. 15(3), 583-607 (2015)

2. Ascanelli, A., Cappiello, M.: Schrödinger-type equations in Gelfand-Shilov spaces. J. Mathématiques Pures et Appliquées 132, 207-250 (2019)

3. Dreher, M.: Necessary conditions for the well-posedness of Schrödinger type equations in Gevrey spaces. Bull. Sci. Math. 127, 485-503 (2003)

4. Ichinose, W.: Some remarks on the Cauchy problem for Schrödinger type equations. Osaka J. Math. 21, 565-581 (1984)

5. Jensen, A.: Commutator method and smoothing property of the Schrödinger evolution group. Math. Z. 191, 53-59 (1986)

6. Kajitani, K.: Smoothing effect in Gevrey classes for Schrödinger equations. Investigations on the structure of solutions to partial differential equations (Japanese) (Kyoto, 1998). Sūrikaisekikenkyūsho Kōkyōroku No. 1056, 46-58 (1998)

7. Kajitani, K., Baba, A.: The Cauchy problem for Schrödinger type equations. Bull. Sci. Math. 119, 459-473 (1995)

8. Kajitani, K., Nishitani, T.: The hyperbolic Cauchy problem, vol. 1505. Lecture Notes in Mathematics, Springer, Berlin (1991)

Publisher's Note Springer Nature remains neutral with regard to jurisdictional claims in published maps and institutional affiliations.

\section{Affiliations}

\author{
Alessia Ascanelli ${ }^{1}$ (D) Massimo Cicognani ${ }^{2} \cdot$ Michael Reissig $^{3}$ \\ Massimo Cicognani \\ massimo.cicognani@unibo.it \\ Michael Reissig \\ reissig@math.tu-freiberg.de \\ 1 Dipartimento di Matematica e Informatica, Università di Ferrara, Via Machiavelli 30, \\ 44121 Ferrara, Italy \\ 2 Dipartimento di Matematica, Università di Bologna, Piazza di Porta San Donato, 5, 40126 Bologna, \\ Italy \\ 3 Faculty for Mathematics and Computer Science, Institute of Applied Analysis, Technical University \\ Bergakademie Freiberg, Prüferstraße 9, 09596 Freiberg, Germany
}

